\title{
Grounding principles for inferring agency: Two cultural perspectives
}

bethany 1. ojalehto, ${ }^{a}$ Douglas L. Medin, ${ }^{a}$ and Salino García G. ${ }^{b}$

aPsychology Department, Northwestern University

2029 Sheridan Road - 102 Swift Hall

Evanston, IL 60208-2710

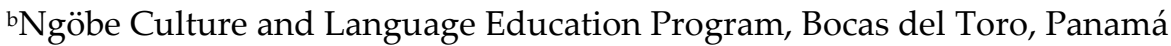

Address correspondence to: bethanyojalehto@u.northwestern.edu

\begin{abstract}
The present research investigates cultural variation in grounding principles for inferring agency in order to address an important theoretical debate: does cultural diversity in agency concepts reflect an animistic overextension of (universal) folkpsychology, as many have argued, or an alternative theory of folkcommunication based on relational principles? In two experiments, mind perception measures were adapted to assess beliefs concerning the agency of non-animal kinds (plants, abiotic kinds, complex artifacts) among Indigenous Ngöbe adults in Panama and US college students. Agency attributions varied systematically, with Ngöbe ascribing greater agency to non-animal natural kinds and US college participants ascribing greater agency to complex artifacts. Analysis of explanations revealed divergent interpretations of agency as a prototypically human capacity requiring consciousness (US), versus a relational capacity expressed in directed interactions (Ngöbe). Converging measures further illuminated the inferential principles underlying these agency attributions. (1) An experimental relational framing of agency probes facilitated Ngöbe but not US agency attributions. (2) Further analysis showed that three key dimensions of agency attribution (experience, cognition, animacy) are organized differently across cultures. (3) A Bayesian approach to cultural consensus modeling confirmed the presence of two distinct consensus models rather than variations on a single (universal) model. Together, these results indicate that conceptual frameworks for agency differ across US college and Ngöbe communities. We conclude that Ngöbe concepts of agency derive from a distinct theory of folkcommunication based on an ecocentric prototype rather than overextensions of an anthropocentric folkpsychology. These observations suggest that folkpsychology and mind perception represent culture specific frameworks for agency, with significant implications for domain-specificity theory and our understanding of cognitive diversity.
\end{abstract}

Keywords: agency; folkpsychology; mind perception; culture; domain specificity; animism 


\section{Introduction}

Agents captivate our attention and animate our worlds. A major goal for cognitive science is to understand how people recognize an agent and conceptualize its capacity to act. These concepts actively structure social cognition and moral reasoning and play an integral role in causal reasoning, folk theories, mind perception, and language (e.g., Banaji \& Gelman, 2013; Carey, 2009; Lowder \& Gordon, 2015; Waytz et al., 2010). Agency concepts are so fundamental that they are often presumed to be universal (Johnson, 2003; Spelke \& Kinzler, 2007; Sperber \& Hirschfeld, 2004). At the same time, it is increasingly clear that agency concepts are multifaceted. Exploring the principles underlying the conceptual structure of the agency domain is the focus of a growing body of research (Gray et al., 2007; Knobe, 2011; Tamir et al., 2016). This literature has overwhelmingly focused on agency as construed through the lens of folkpsychology where agency is intimately linked to the inference of having a mind. It has also overwhelmingly focused on Western study samples. While folkpsychology may be fundamental to Western conceptions of agency, we believe there is striking cultural variation in grounding principles for inferring agency. Specifically, we propose that agency can be conceptualized as folkcommunication rather than folkpsychology, and consequently that important features of agency attribution, long assumed to be universal, may vary systematically across cultures. Here we report one such case, contrasting conceptual frameworks for agency between Indigenous Ngöbe of Panama and US college students.

\section{Background}

Agency, broadly defined as the capacity to act, is a signature property of moving, living, and sentient kinds. Depending on the interpretive stance adopted, an act of agency may be conceptualized quite differently depending on whether it is thought to arise from purely physical or biological mechanisms versus more complex psychological processes (e.g., Strickland et al., 2016). For instance, individuals may interpret movement toward the sun differently for a cloud versus a plant (physical versus biological), and both differently from a person's movement toward the sun (psychological) (Gutheil et al., 1998; Opfer \& Gelman, 2001; Opfer \& Siegler, 2004). How people parse these conceptual categories for agency is an important question for cognitive science (Csibra \& Gergely, 2007; Lewis, 1990). The influential domain-specificity framework theory proposes that people intuitively understand action in terms of (at least) three distinct folktheories corresponding to specific ontological kinds: the force dynamics of physical kinds (folkphysics), the teleological agency of living kinds (folkbiology), or the mental agency of animate kinds based on humans as the prototypical agent (folkpsychology) (Wellman \& Gelman, 1992).

Within the folkpsychological domain, a further distinction has been proposed between two forms of mental agency associated with either experiential or cognitive capacities (Gray et al., 2007). These two dimensions of mind perception are thought to involve distinct inferences about biological versus representational faculties, respectively (Knobe, 2011). Among Western samples, for example, humancreated entities such as artificial intelligence or corporations are judged high on cognition but low on experience (as they are not alive), whereas nonhuman animals are judged high on experience but low on cognition (as they are not considered to have conscious representations). This seems to reflect an iteration of the broader folkbiology-folkpsychology distinction within the domain of psychology proper.

Folkpsychology is widely seen as a universal intuitive framework theory for agency (Gelman \& Legare, 2011; Sperber \& Hirschfeld, 2004), despite the fact that it follows from a theoretical partitioning of cognitive domains that has been developed primarily among Western cultures by Western researchers. At the same time, it is well known that cultures diverge in explicit beliefs about agency. For instance, many Indigenous cultures hold that non-animal natural kinds like plants, minerals, celestial bodies and abiotic forces (e.g., thunder, ocean) may perceive, respond, and communicate (Hallowell, 1960; Harvey, 2005). These are capacities that most Westerners, researchers included, view as psychological ones. As 
such, proponents of domain-specificity theory have treated these cultural beliefs as overextensions of a universal folkpsychology beyond its proper domain (Atran \& Norenzayan, 2004; Boyer, 1996). These overextension accounts explain Indigenous understandings of nonhuman agency in terms of counterintuitive, supernatural, or animistic concepts (Barrett, 2000; Boyer, 2003; see also Guthrie et al., 1980) (but see Bird-David, 1999; Ingold, 2006; Kohn, 2007). A related class of explanations characterizes Indigenous agency attributions as metaphorical. Here, the assumption is again that the universal base for inferring agency is a human-like mind, such that attributions of agency to nonhuman targets are metaphors that map "back" to the folkpsychology source domain.

An alternative possibility is that presumed overextensions of folkpsychology instead reflect different cultural concepts of agency stemming from diverse principles for inferring agency, and/or different notions of what belongs in the agency domain. Current theories of folkpsychology in the West emphasize human minds and consequently these theories tend to focus on anthropocentric forms of agency. As an alternative, one framework that has been extensively discussed in the literature on Indigenous epistemologies is a folktheory where agency is primarily understood as a capacity for relational interaction with other agents and environments (Bird-David, 1999; Danziger \& Rumsey, 2013; Harvey, 2005; Kohn, 2013). Working from multiple disciplines and Native perspectives, scholars have argued that Indigenous theories afford recognition of nonhuman agency as it is expressed in diverse ways across the natural world (Kimmerer, 2013; Pierotti, 2011), and that these theories do not share the overtly mentalistic emphasis of Western folkpsychology (e.g., Ingold, 2011; Walker, 2013). These folktheories of agency can be understood in the broader context of Indigenous relational epistemologies, which are cultural framework theories that emphasize the interconnectedness of all things and include nonhumans as part of the social community (Medin et al., 2013; Pierotti, 2011). ${ }^{1}$ This raises the possibility that concepts of agency may be organized differently across cultures.

Building on these observations, we propose that cultural variability in conceptual frameworks for agency can be distinguished along (at least) two dimensions: (a) distinct ecocentric or anthropocentric prototypes for agency, following from a focus on natural actors broadly or humans specifically; and (b) distinct causal theories of agency as a relational capacity (reasoning about interactions) or a psychological capacity (reasoning about representational mental states). On this account, people may attend to similar basic cues for agency perception across cultures (e.g., Barrett et al., 2005), but those cues acquire very different meanings across cultural frameworks. Our current studies, then, do not ask whether people detect agency differently but whether they infer capacities differently given that agency has been detected.

Our research focuses on the Indigenous Ngöbe of Bocas del Toro, Panama. The primary research community is located on a heavily forested island in the Caribbean that forms a political annex to the Indigenous autonomous region Comarca Ngöbe-Bugle. The community hosts about 600 people in several dispersed family hamlets that lie within an hour's walking or canoeing distance of each other. Community members' lifestyles are closely coordinated with the surrounding ecology through agroforestry, fishing and diving, and other subsistence practices (Gordon, 1982). Our prior research findings (ojalehto et al., 2017; ojalehto et al., 2015; ojalehto et al., 2013) indicate that Ngöbe attend to nonhuman agency and ecological relationships in ways that resonate with Indigenous relational

\footnotetext{
${ }^{1}$ Indigenous relational epistemologies bear similarities to work on Eastern collectivist cultures where holistic cognitive styles also emphasize relationality (Nisbett et al., 2001). However, holistic cognitive styles are broad enough to encompass anthropocentric folkpsychology (e.g., Inagaki \& Sugiyama, 1988) and need not be ecological or even attentive to communication. By contrast, Indigenous relational epistemologies are explicit about the role of nonhuman plants, animals, and natural forces as agents who engage in communicative relationships (e.g., Harvey, 2005; Kohn, 2013). Nonetheless, both cultural orientations call attention to the relative lack of emphasis on relationships in Western thought, underscoring the need for cognitive science to go beyond Western samples.
} 
epistemologies more generally. This is corroborated by our own experience as a community member (S.G.G.) and long-term researcher in the community (over seven years of regular fieldwork by b.l.o.). As we will see, Ngöbe informants focus on different dimensions of agency across a different scope of actors than would be predicted by current accounts of folkpsychology. From this we argue that grounding principles for inferring agency vary across cultures, leading to folktheories that track distinctive domains of agency: Ngöbe take a communicative stance on agency as a relational capacity to dynamically interact with the world, whereas US individuals take a psychological stance on agency as an individual capacity to internally represent the world. These findings make original contributions to the cognitive study of agency and animism by revealing the cultural boundaries of folkpsychology, presenting an alternative folktheory of communication, and presenting a new interpretation of animism.

\section{Current research}

In two experiments, we adapted the mind perception survey (Gray, et al., 2007) to assess concepts of agency among US college students and Indigenous Ngöbe adults of Panama. The key hypotheses center on concepts of agency for non-animal kinds: specifically, plants, abiotic kinds (i.e., nonliving natural kinds), ${ }^{2}$ and complex computerized artifacts (henceforth referred to simply as complex artifacts). We predicted that Ngöbe participants would attribute greater agency to plants and abiotic kinds relative to complex artifacts (and as compared to US college participants), on the basis of those natural entities' capacity to interact with the environment. On an anthropocentric prototype, we predicted the opposite: namely, that US college participants would recognize greater agency for complex artifacts relative to plants and abiotic kinds (and as compared to Ngöbe), due to their human-designed information processing capacities.

Experiment 1 has two main goals. First, we sought to establish whether there are cultural differences in attribution of agency capacities to various natural and artifact non-animal kinds. ${ }^{3}$ The second goal was to assess conceptual frameworks by analyzing participants' explanations for their agency attributions. We predicted that Ngöbe and US respondents would draw on different framings of and criteria for the capacities under question, corresponding to distinct folktheories of agency.

Experiment 2 aimed to replicate the findings from Experiment 1 with larger samples and to provide additional insight into the grounds for inferring agency on each cultural framework. First, an experimental manipulation used relational framings of agency probes in order to assess the proposed role of relational principles on the Ngöbe folkcommunication framework. Second, additional probes were included to explore the cultural organization of conceptual dimensions relevant to agency concepts, including cognition, experience, and animacy. Last, we used recent advances in cultural consensus modeling (Anders \& Batchelder, 2012; Anders et al., 2014) to detect whether the observed cultural differences reflect multiple latent conceptual models or variations on a single (universal) model, and to identify the conceptual features and individual-level differences associated with each model.

\section{Experiment 1}

\subsection{Participants}

Participants in Experiment 1 were Indigenous Ngöbe adults of Panama $(n=11 ; 3$ female) and US college students $(n=11 ; 4$ female).

\footnotetext{
${ }^{2}$ Entities such as the sun, ocean, or rocks are often referred to as nonliving natural kinds in the Western literature, but we refer instead to abiotic kinds (defined by Merriam Webster as "not biological; especially: not involving or produced by organisms") because many Ngöbe individuals consider these kinds to be alive, as we will report.

${ }^{3}$ These capacities (e.g., thought, communication) are typically characterized as mental capacities, but we refer instead to agency capacities because the specific nature of these conceptual commitments is in question.
} 
Ngöbe context. Ngöbe participants' ages ranged from 22-63 years $(M=34.73, S D=12.42)$. Experience with formal schooling ranged from 0-12 years $(M=6.82$; $S D=3.09)$. Older informants tended to have less formal schooling, $(r=-.17, p=.21)$ but this did not reach reliability. Ngöbe participants were bilingual in Spanish and their native Ngöbere. Participants were recruited through household visits or community meetings. Community permission and individual informed consent were received. Ngöbe participants received a small compensation and a donation was made to the community fund.

Everyday life in the primary Ngöbe research community reflects a dynamic economic and environmental context. Many of the major lifestyle changes of recent decades reflect accelerating tourism and real estate development on or near Ngöbe lands, particularly in areas that, like our primary research community, are annexed to the Comarca (Mayhew et al., 2010). Although Ngöbe gained recognition of the semi-autonomous Comarca Ngöbe-Bugle territory in 1997, this area represents half their traditional territory (Young \& Bort, 1999) and encompasses only about two-thirds of the Ngöbe population (Mayhew, et al., 2010). Today, Ngöbe communities in Panama face major environmental issues stemming from protracted struggles against copper mines, hydroelectric dams, and other forms of natural resource development on Ngöbe lands (Gjording, 1991).

In the primary research community, subsistence activities are increasingly complemented by wage labor (primarily eco-tourism related) and other forms of market integration including consumption of store bought food and sundries and the small-scale sale of crops, fish harvests, forest products, and handicrafts. Daily life involves light reliance on infrastructure. While ecologically integrated infrastructure such as mangrove canals and forest paths aid transport, there are no roads, centralized electricity, or running water. Households rely on nearby streams for water, generator/battery-operated or traditional tools for appliances, and travel by foot or canoe for local transport. There are several public and family-owned motorboats for transport to and from hubs on the mainland and Bocas Island. Despite the lack of a centralized electricity source, certain forms of technology have become household staples in recent years. Cell phones and radios are now ubiquitous in the community while televisions and computers are increasingly common. For example, a recent Panamanian policy allocated laptop computers to all secondary school students, which effectively introduced the personal computer to a majority of Ngöbe households.

Ecological relationships have been and remain integral to Ngöbe lifestyles. This is particularly evident in the local Indigenous nativistic church, which represents a cultural revitalization movement for Ngöbe philosophy and practices (Young, 1975). Religious practice includes observances of ecological phenomena such as lunar events and harvest rituals, which are celebrated in church meetings that present traditional oral histories and contemporary teachings in the native Ngöbere language (see also Montezuma, 1991; Reid, 1994; Sarsaneda del Cid, 2009). Despite the centrality of this ecological relationship, Ngöbe lands have increasingly become a site of cultural conflict over environmental management. The primary research community is located in a sensitive and biodiverse archipelago ecosystem, and large areas of land adjacent to the research community were converted to national park in the 1980s. Many Ngöbe subsistence activities are now prohibited on these lands despite the fact that these forests are anthropogenic and Indigenous practices enrich rather than deplete biodiversity (Bray et al., 2004; Gordon, 1982). Ngöbe community members negotiate similar tensions with regard to laws surrounding hunting and harvesting of endangered species.

US context. US participants were undergraduate students enrolled in an introductory psychology course at Northwestern University in the Greater Chicago Area. Ages ranged from 18-21 years $(M=19.45$, $S D=1.21$ ). All participants were fluent English speakers. The students came from predominantly uppermiddle class backgrounds (60\%); and identified as Caucasian (57\%), Asian/Asian-American (22\%), Black/African-American (7\%), Latino/Latina (3\%) or other (12\%). US participants completed informed consent and received partial course credit for participating. 


\subsection{Methods}

As part of a larger interview (identical for both groups), participants completed an adapted version of the mind perception survey (Grey et al., 2007) (see Appendix A1). Participants rank ordered 16 entities in terms of their capacity for several kinds of agency, allowing for ties (indicating equal capacity) and exclusions (indicating no capacity). Five representative capacities were selected from the original 18item mind perception survey: thought, communication, morality, hunger and desire. ${ }^{4}$ Each participant ranked (on average) only three of the five capacities to keep interviews of reasonable duration. The particular capacities presented to each participant were randomly selected.

Participants were probed to explain a subset of their rankings. Ngöbe and US respondents provided a similar number and length of explanations for each capacity and kind (for details, see Appendix A4).

Stimuli were cards with color photographs of individual entities, and included 4 humans (old woman, young woman, infant, fetus), 3 animals (chimp, dog, cow), 2 plants (banana, manioc [Panama]; lettuce, strawberry [US]), 5 abiotic kinds (sun, clouds, rain, ocean, rocks), and 2 complex artifacts (robot, computer). Notably, previous mind perception surveys have rarely included plants or abiotic kinds, preferring instead computerized artifacts; this may itself be a commentary on Western views of agency.

Participants were interviewed in Spanish (Panama) or English (US). The Spanish protocols were independently forward- and back-translated by two trained research assistants in the US. Before use in Panama, Spanish protocols checked for local validity by a trilingual (in Spanish, English, and Ngöbere) researcher (S.G.G.). All interviews were audio-recorded and transcribed, and Spanish responses were translated into English for coding analysis.

\subsection{Agency attributions}

\subsubsection{Data analysis}

We used a weighted rank system to calculate mean ranks, with ranks assigned by level and lower numbers indicating higher rank (range: 1-16). Ties were treated as the median score across that level (e.g., if two items were tied for the first level, each received a score of 1.5), and excluded items were scored as tied for last place (e.g., if two items were excluded, each received a score of 15.5).

Agency attribution profiles. Cultural models can be inferred by comparing how non-animal kinds (plants, abiotic kinds, and complex artifacts) are ordered relative to one another within each cultural group. These profiles could fall under one of two general models. On a hypothesized ecocentric prototype, Ngöbe responses should follow a natural kinds model (NKM) where both plants and abiotic kinds are included more frequently and ranked above complex artifacts. On a hypothesized anthropocentric prototype, US responses should follow an artifact kinds model (AKM) where complex artifacts are included more frequently and ranked above natural kinds, either abiotic kinds or both plants and abiotic kinds (depending on the mind perception dimension).

We did not predict marked cultural differences for animals and humans, or for mammals versus non-mammals, on these particular measures. Ngöbe and US individuals may in fact take distinct views of animal agency, particularly for complex capacities such as morality as we have found in other studies (ojalehto et al., 2015). But the present measures were aimed at discriminating coarse differences across the animal/non-animal divide rather than subtle distinctions in degree of capacity accorded to any one kind. ${ }^{5}$

${ }^{4}$ The five probes were selected to include capacities associated with both mind perception dimensions, but specifically probing these two dimensions was not the goal of Experiment 1 as it was in Experiment 2.

${ }^{5}$ On an anthropocentric prototype, one might also predict that US participants would rank humans relatively higher, and mammals relatively higher than non-mammals, than Ngöbe participants. Assessing these possibilities would require an interval measure of perceived distance between human, mammal, and non-mammal capacities, such as a rating scale. The present measures of ordinal ranks and inclusion rates are not sensitive to such differences. 
Table 1. Mean rankings and percent inclusions for agency ${ }^{\mathrm{a}}$

\begin{tabular}{|c|c|c|c|c|c|c|c|c|c|c|c|c|c|c|}
\hline \multirow[b]{3}{*}{ Kind } & \multicolumn{7}{|c|}{ Mean Percent Inclusions } & \multicolumn{7}{|c|}{ Mean Ranks } \\
\hline & \multicolumn{2}{|c|}{$\begin{array}{l}\text { Ngöbe } \\
(n=11)\end{array}$} & \multicolumn{2}{|c|}{$\begin{array}{c}\text { US } \\
(n=11)\end{array}$} & \multirow[b]{2}{*}{$t$-test } & \multicolumn{2}{|c|}{$\begin{array}{l}\text { Overall } \\
(N=22)\end{array}$} & \multicolumn{2}{|c|}{$\begin{array}{l}\text { Ngöbe } \\
(n=11)\end{array}$} & \multicolumn{2}{|c|}{$\begin{array}{c}\text { US } \\
(n=11)\end{array}$} & \multirow[b]{2}{*}{$U$-test } & \multicolumn{2}{|c|}{$\begin{array}{l}\text { Overall } \\
(N=22)\end{array}$} \\
\hline & $M$ & $S D$ & $M$ & $S D$ & & $M$ & $S D$ & $M$ & $S D$ & $M$ & $S D$ & & $M$ & $S D$ \\
\hline Humans & $0.93_{a}$ & $(0.10)$ & $0.85 a$ & $(0.14)$ & $n s$ & $0.89 \mathrm{a}$ & $(0.12)$ & $3.86_{a}$ & $(1.58)$ & $4.21_{\mathrm{a}}$ & $(.58)$ & ns & $4.03 \mathrm{a}$ & $(1.18)$ \\
\hline Animals & $1.00_{\mathrm{a}}$ & $(0.00)$ & $0.83_{a}$ & $(0.21)$ & $2.79^{*}$ & $0.91_{\mathrm{a}}$ & $(0.17)$ & $6.51_{\mathrm{b}}$ & $(1.94)$ & $5.66_{b}$ & $(1.16)$ & ns & $6.08_{\mathrm{b}}$ & $(1.62)$ \\
\hline Plants & $0.55_{b}$ & $(0.33)$ & $0.21_{\mathrm{b}}$ & $(0.26)$ & $2.72^{* *}$ & $0.38_{b}$ & $(0.34)$ & $10.65_{c}$ & $(1.23)$ & $11.08_{\mathrm{c}, \mathrm{d}}$ & $(1.19)$ & ns & $10.87_{c}$ & $(1.20)$ \\
\hline Abiotic kinds & $0.45_{b}$ & $(0.37)$ & $0.08_{b}$ & $(0.17)$ & $3.04^{* *}$ & $0.26_{b}$ & $(0.34)$ & $11.00_{\mathrm{c}}$ & $(1.71)$ & $11.78_{\mathrm{d}}$ & $(0.85)$ & ns & $11.39_{c}$ & $(1.38)$ \\
\hline Complex artifacts & $0.38_{b}$ & $(0.36)$ & $0.29_{b}$ & $(0.26)$ & $n s$ & $0.34_{b}$ & $(0.31)$ & $12.35_{\mathrm{c}}$ & $(1.55)$ & $10.58_{\mathrm{c}}$ & $(1.40)$ & $25.0^{*}$ & $11.47_{\mathrm{c}}$ & (1.70) \\
\hline
\end{tabular}

${ }^{a}$ Agency is a composite measure of thought, communication, morality, hunger, and desire.

${ }^{b}$ Mean ranks: Lower numbers indicate higher ranks (range: 1 - 16); with excluded items scored as tied for last place.

${ }^{\mathrm{c}}$ Main effect for kind: Means in the same column not sharing the same subscript differ significantly at $p<.05$ in Wilcoxon signed-rank tests.

Note: ${ }^{*}$ Indicates $p<.05 ;{ }^{* *} p<.01 ;{ }^{* *} p<.001$.

\subsubsection{Results}

Our main predictions concerning culturally distinct frameworks for nonhuman agency were borne out, as summarized in Table 1. Here we focus on results pertaining to the hypothesized cultural differences (see Table 1; for other detailed results see Appendix A2.) We analyzed results by considering which entities were included in agency rankings (inclusion) and by their mean rank (ranking). ${ }^{6}$ Each measure was averaged by kind across the five agency capacities, then analyzed with a 2 (culture) X 5 (kind: artifact, abiotic kind, plant, animal, human) repeated-measures ANOVA. Degrees of freedom were corrected using Huynh-Feldt estimates due to violation of assumption of sphericity (inclusion: $\chi^{2}(9)=$ 28.31, $p<.01, \varepsilon>.75$; ranking: $\left.\chi^{2}(9)=18.02, p<.05, \varepsilon>.75\right)$.

Inclusions. Agency inclusions for each kind differed substantially across cultures, $F(3.24,64.87)=$ $2.86, p<.05, \eta 2=.13$. As predicted on an ecocentric prototype, Ngöbe were more likely than US college participants to include non-animal natural kinds in their agency ranks, including both plants (Ngöbe: $M=$ $0.55, S D=0.33$; US: $M=0.21, S D=0.26$ ), and abiotic kinds (Ngöbe: $M=0.45, S D=0.37$; US: $M=0.08, S D=$ 0.17 ), as well as animals (Ngöbe: $M=1.00, S D=0$; US: $M=0.83, S D=0.21$ ), $p s<.05$. However, the two groups did not differ reliably on inclusion of complex artifacts. Thus, our prediction that US participants would be more likely to include complex artifacts on an anthropocentric prototype was not borne out.

Rankings. We considered agency attribution profiles by analyzing differences in mean ranks for each non-animal kind across cultures, as well as differences in the mean rank ordering of those kinds within each cultural group. Mean ranks varied by kind across the two groups, $F(3.47,69.35)=2.70, p<.05$, $\eta 2=.12$. Follow-up comparisons using the Mann-Whitney $\mathrm{U}$ test confirmed that US college rankings were significantly higher than Ngöbe adult rankings for complex artifacts (US: $M=10.58, S D=1.40$; Ngöbe: $M$ $=12.35, S D=1.55), U=25, p<.05$. Ngöbe tended to rank plants and abiotic kinds higher than US participants, but these differences did not reach reliability.

Within-culture pair-wise rank comparisons using the Wilcoxon signed-rank test revealed modest differences between non-animal kinds consistent with the hypothesized agency frameworks, although not all the predicted differences reached reliability. Ngöbe followed the predicted natural kinds model by tending to rank plants and abiotic kinds above complex artifacts, and the higher rank for plants relative to complex artifacts was marginally significant $(Z=-1.89, p=.06)$. The opposite held for the US rank

\footnotetext{
${ }^{6}$ Because exclusions were scored as tied for last place, mean rank is insensitive to differences between items that were excluded and those included but ranked last. For this reason it is most straightforward to assess cultural differences in terms of differences in inclusion profiles, as we do in the first three sections of Experiment 2 results.
} 
Table 2: Coding categories related to anthropocentric psychological agency

\begin{tabular}{|c|c|c|}
\hline Code & Description & Examples \\
\hline $\begin{array}{l}\text { Human- } \\
\text { centric } \\
\text { framing }\end{array}$ & $\begin{array}{l}\text { Implicates humans as prototypical } \\
\text { agents by referring to: } \\
\text { (i) Human-nonhuman comparisons } \\
\text { (ii) Human perception of agency } \\
\text { (iii) Human intervention on agency }\end{array}$ & $\begin{array}{l}\text { (i) Animals "don't have the capacity to think like people" (NG, } \\
\text { Th.) } \\
\text { (ii) Animals "not so much, because they're difficult to interpret" } \\
\text { (US, Com.) } \\
\text { (iii) Dogs have "been conditioned to act that way" (US, Mor.) }\end{array}$ \\
\hline Scalar framing & $\begin{array}{l}\text { Frames agency as scalar capacity by: } \\
\text { (i) Assessing agency in terms of } \\
\text { hierarchical scales or timelines } \\
\text { (ii) Hedging the sense in which an } \\
\text { entity has capacity, implying an ideal }\end{array}$ & $\begin{array}{l}\text { (i) Mammals "tend to be smarter, have a more complex brain" } \\
\text { (US, Th.); Baby will "given time, overcome the chimpanzee" (US, } \\
\text { Mor.) } \\
\text { (ii) Plants "feel emotion in like a different sense" (US, Com.); } \\
\text { "But the plant doesn't really get hungry" (NG, Hun.) }\end{array}$ \\
\hline $\begin{array}{l}\text { Internalized } \\
\text { indicators }\end{array}$ & $\begin{array}{l}\text { Focus on internal parts or substrates } \\
\text { underlying indicators of agency: } \\
\text { (i) mind or brain } \\
\text { (ii) interior or bodily substrates }\end{array}$ & $\begin{array}{l}\text { (i) Animals "have receptors and stuff in their brain that signal } \\
\text { when they're hungry" (US, Hun.) } \\
\text { (ii) Robot has "electric cables in their body" (NG, Com.); Human } \\
\text { "body requires nutrients" (US, Hun.) }\end{array}$ \\
\hline Consciousness & $\begin{array}{l}\text { Consciousness is criterial to agency, } \\
\text { as indicated by: } \\
\text { (i) self-awareness } \\
\text { (ii) autonomy over own actions } \\
\text { (iii) (not) instincts or mere reactions }\end{array}$ & $\begin{array}{l}\text { (i) Plants "perform actions" but "have no concept of being } \\
\text { moral" (US, Mor.); Baby “can't recognize" its hope (US, Des.) } \\
\text { (ii) Animal's "brain doesn't control what he's communicating" } \\
\text { (US, Com.) } \\
\text { (iii) Cows have "primal instinct rather than...more technical } \\
\text { cognition levels" (US, Th.) }\end{array}$ \\
\hline
\end{tabular}

Abbreviations: (NG/US) Ngöbe/US; (Hun.) Hunger; (Th.) Thought; (Mor.) Morality; (Des.) Desire; (Com.) Communication

profile, which followed an artifact kinds model where complex artifacts were ranked significantly higher than abiotic kinds $(Z=-2.03, p<.05)$ but not reliably higher than plants.

\subsection{Interim summary}

So far, US attribution profiles are consistent with a view of psychological agency as a property primarily restricted to humans and animals, whereas Ngöbe profiles align with a more ecologically oriented view of agency as a capacity expressed by many natural kinds including plants and abiotic kinds. The inferential principles at stake in these cultural frameworks for agency were further investigated by analyzing participants' explanations for their agency rankings.

\subsection{Explanatory frameworks for agency}

An entity can express agency in many ways. Whether those expressions are interpreted as relevant to a particular capacity hinges on one's conception of agency. We assessed cultural stances on this matter by analyzing participants' explanations for their agency rankings.

Building on previous accounts of Western and Indigenous cultural epistemologies (see Medin et al., 2013), as well as the (predominantly Western) literature on folkpsychology and mind perception, we developed a coding scheme to assess constructs associated with each hypothesized cultural framework.

Our first hypothesis holds that conceptual frameworks for agency are structured around different prototypes, which we expect will correspond to distinct framings of agency. If US folkpsychology treats humans as the prototype, this should be linked to a scalae naturae framing where organisms are hierarchically ordered on a scale from simple to complex human agency. In contrast, Ngöbe are predicted to frame agency as a capacity for relating that takes many forms and is exercised by many human and nonhuman entities. Accordingly, we predicted that US explanations would contain more human-centric and scalar framings of agency, while Ngöbe explanations would contain more relational framings of agency.

Our second hypothesis holds that cultural frameworks take different stances on the relevant causal principles for inferring agency, either, as an interactive or a psychological phenomenon. 
Table 3: Coding categories related to ecocentric relational agency

\begin{tabular}{|c|c|c|}
\hline Code & Description & Examples \\
\hline $\begin{array}{l}\text { Relational } \\
\text { framing }\end{array}$ & $\begin{array}{l}\text { Frames agency as relational capacity } \\
\text { by referring to interactions and } \\
\text { other-orientated states involving: } \\
\text { (i) other agents } \\
\text { (ii) environments }\end{array}$ & $\begin{array}{l}\text { (i) Chimps "have a good sense of social structure" (US, Th.); } \\
\text { Cows "know their owner" but are "fierce" to others (NG, Mor.) } \\
\text { (ii) "Plants have hunger, for the rain that falls" (NG, Hun.); Sun } \\
\text { "communicates with [water] in the moment of rising" (NG, } \\
\text { Com.) }\end{array}$ \\
\hline $\begin{array}{l}\text { Interactive } \\
\text { indicators }\end{array}$ & $\begin{array}{l}\text { Focus on observable interactions as } \\
\text { cues to agency, including: } \\
\text { (i) Behavioral patterns } \\
\text { (ii) Means of expressing agency }\end{array}$ & $\begin{array}{l}\text { (i) Dogs have "certain things that they do or don't do, when they } \\
\text { live with people" (US, Mor.) } \\
\text { (ii) Plants "communicate in the way they go growing" (NG, } \\
\text { Com.); Animals "have their distinct forms to wait, express, } \\
\text { know" (NG, Des.) }\end{array}$ \\
\hline Directedness & $\begin{array}{l}\text { Directedness is criterial to agency, } \\
\text { as indicated by: } \\
\text { (i) goal-directed needs or wants } \\
\text { (ii) teleological processes } \\
\text { (iii) variable states of the entity }\end{array}$ & $\begin{array}{l}\text { (i) Plants are "hungry for something that will allow them to } \\
\text { survive" (US, Hun.) } \\
\text { (ii) Sun "has the thought to light the world" (NG, Th.); } \\
\text { Rain "has thoughts, that it falls as the rain" (NG, Th.) } \\
\text { (iii) Oceans "have a moment where they wait for the change" } \\
\text { (NG, Des.) }\end{array}$ \\
\hline
\end{tabular}

Abbreviations: (NG/US) Ngöbe/US; (Hun.) Hunger; (Th.) Thought; (Mor.) Morality; (Des.) Desire; (Com.) Communication

Accordingly, Ngöbe and US explanations are expected to focus on different indicators of and criteria for ascribing agency.

\subsubsection{Coding scheme}

All explanations were analyzed with a coding scheme designed to assess framings of and explanatory constructs for agency on both hypothesized cultural frameworks (see Tables 2 and 3). Our coding system measured the extent to which participants' explanations focused on the following variables: human-centric, scalar, or relational framings of agency; internalized or interactive indicators of agency; and consciousness or directedness as criteria for agency. (Each is detailed below.) Reference to a construct of interest (e.g., brain) was coded whether it was invoked for agency endorsement (e.g., "chimps can think because they have a brain") or denial (e.g., "plants cannot think because they don't have a brain"). Coding categories were not mutually exclusive.

The dependent variable was the percent of explanatory content associated with a given coding category (number of codes divided by total words in explanation, times 100).

Two raters (b.o. and a US research assistant blind to the hypotheses) each coded half the data independently, after working together to iteratively develop and train on the coding scheme. Inter-rater agreement was good, with intra-class correlations for each coding variable ranging from $r=.62$ to $r=.85$, all $p s<.01,(d f=232)$ (see Appendix A3, Table A1). To ensure the most accurate code assignment possible, both raters separately coded $90 \%$ of explanations (including those used to establish reliability), disagreements were discussed, and the consensus decision was used for the final data set. The remaining $3 \%$ and $6 \%$ of explanations were coded independently by each of the two (equally expert) raters.

\subsubsection{Results}

The results roundly supported the proposal for two distinct conceptual frameworks, as confirmed by significant cultural differences observed across the majority of coding categories (summarized in Table 4). All tests reported below are 2 (culture) x 2 (kind: animate versus inanimate, as defined on Western categories) repeated-measures ANOVAs on the coding variable of interest. Data were collapsed over the five kinds into two categories (in/animate) to reduce empty cells, given that not all participants provided specific explanations for each kind on every ranking (this split allowed us to include 10 of 11 participants 
Table 4. Explanatory Content Associated with Coding Constructs by Culture

\begin{tabular}{|c|c|c|c|c|c|c|c|}
\hline \multirow[b]{3}{*}{ Agency Framework } & \multirow[b]{3}{*}{ Coding construct } & \multicolumn{6}{|c|}{ Percent Explanatory Content (across kinds) } \\
\hline & & \multicolumn{2}{|c|}{ US } & \multicolumn{2}{|c|}{ Ngöbe } & \multicolumn{2}{|c|}{ Overall } \\
\hline & & $M$ & $S D$ & $M$ & $S D$ & $M$ & $S D$ \\
\hline \multirow[t]{4}{*}{$\begin{array}{l}\text { Anthropocentric \& psychological } \\
\text { (US college student model) }\end{array}$} & $\begin{array}{l}\text { Human-centric } \\
\text { framing }\end{array}$ & $2.66^{*}$ & $(1.93)$ & $0.78^{*}$ & $(0.63)$ & 1.72 & $(1.70)$ \\
\hline & Scalar framing & $6.22 *$ & $(2.54)$ & $2.71 *$ & $(3.06)$ & 4.46 & 3.28 \\
\hline & $\begin{array}{l}\text { Internalized } \\
\text { indicators }\end{array}$ & 0.53 & $(0.50)$ & 0.91 & $(2.62)$ & 0.72 & $(1.84)$ \\
\hline & Consciousness & $1.31 *$ & $(0.92)$ & $0.24 *$ & $(0.47)$ & 0.78 & $(0.89)$ \\
\hline \multirow{3}{*}{$\begin{array}{l}\text { Ecocentric \& relational } \\
\text { (Ngöbe adult model) }\end{array}$} & Relational framing & $3.82 *$ & $(2.37)$ & $7.47^{*}$ & $(3.41)$ & 5.65 & $(3.42)$ \\
\hline & $\begin{array}{l}\text { Interactive } \\
\text { indicators }\end{array}$ & $0.60 *$ & $(0.85)$ & $1.78^{*}$ & $(0.91)$ & 1.19 & $(1.05)$ \\
\hline & Directedness & $0.93 *$ & $(0.96)$ & $4.32 *$ & $(1.92)$ & 2.62 & $(2.28)$ \\
\hline Overall content coded & & $16.07 \%$ & $(3.43 \%)$ & $18.20 \%$ & $(5.10 \%)$ & $17.14 \%$ & $(4.36 \%)$ \\
\hline
\end{tabular}

from each cultural group). Here we report only results that reached statistical significance (see Appendix A4 for detailed results). ${ }^{7}$

\section{(i) Human-centric framing of agency}

On an anthropocentric model, we predicted that US explanations would treat humans as the prototype and thus contain more human-centric framings of agency. An anthropocentric framing was assessed by coding for (i) comparison of nonhumans to humans; (ii) taking a human vantage point on perceiving or appraising nonhuman agency; or (iii) human intervention on nonhuman agency. (For examples of these and all subsequent coding categories, refer to Tables 2 and 3.)

As predicted, there was a main effect for culture, $F(1,18)=8.65, p<.01, \eta 2=.33$, such that US college participants provided substantially more human-centric content $(M=2.66 \%, S D=1.93 \%)$ than Ngöbe adult participants $(M=0.78 \% S D=0.63 \%)$.

\section{(ii) Scalar framing of agency}

Following from an anthropocentric prototype, we also predicted that US explanations would treat humans as the most complex or "developed" agents, and thus contain more scalar framings of agency. A scalar framing was defined as (i) assessing agents according to hierarchical taxonomies or developmental scales; or (ii) hedging the sense in which an entity possesses a capacity (e.g., in an "abstract" or "different" sense), implying an anchoring concept based on a prototypical (presumably human) agent. Note that the hedging coding category also captures explicitly metaphorical constructions of agency. Accounts that explain Ngöbe attributions to nonhuman agents as metaphorical overextensions of folkpsychology from a human source would predict greater reliance on this coding category among Ngöbe participants.

The predicted main effect for culture was reliable, $F(1,18)=7.82, p<.05, \eta 2=.30$, such that US college student explanations relied much more on scalar framings $(M=6.22 \%, S D=2.54 \%)$ than $\mathrm{Ngöbe}$ adult explanations $(M=2.71 \%, S D=3.06 \%)$. The low incidence of anthropocentric framings and hedging among Ngöbe informants suggests that their agency attributions do not reflect metaphorical constructions.

(iii) Relational framing of agency

On an ecocentric model, we predicted that Ngöbe explanations would frame agency in terms of relational action, both social and ecological. A relational framing was assessed by coding references to relationships, interactions, and other-oriented states involving (i) other agents (social relations); and (ii) environments (ecological relations).

\footnotetext{
${ }^{7}$ There were no main effects for kind (in/animate) or interactions of culture by kind for any coding outcome variable.
} 
As expected, there was a main effect for culture, $F(1,18)=7.76, p<.05, \eta 2=.30$, such that Ngöbe adult participants provided almost twice as many relational framings $(M=7.47 \%, S D=3.41 \%)$ than US college participants $(M=3.82 \%, S D=2.37 \%)$. The same cultural trends held for both social and ecological relations separately, according to kind (see Appendix A4).

(iv) Indicators of agency

Following from a focus on psychological capacities linked to having a mind, we predicted that US explanations would focus more on internalized indicators of agency by referring to (i) minds and brains, or (ii) other interior substrates (e.g., cables in a robot) that underlie such capabilities. Contrary to our prediction, there was no main effect for culture on explanatory content associated with internalized indicators, $F(1,18)=.20$, ns, (US: $M=.53 \%, S D=.50 \%$; Ngöbe: $M=.91 \%, S D=2.62 \%$ ).

In contrast, we expected Ngöbe explanations to focus on interactive indicators of agency by referring to (i) observable behavioral patterns as evidence of a capacity, or (ii) tangible means and multiple ways of expressing a capacity. As predicted, there was a significant main effect for culture, $F(1,18)=9.01, p<.01, \eta 2=.33$, such that Ngöbe participants focused more on interactive indicators $(M=$ $1.78 \%, S D=.91 \%)$ than US participants $(M=0.60 \%, S D=.85 \%)$.

(v) Criteria for agency

If agency is understood as a psychological capacity as we predict for US participants, then we would expect them to treat consciousness as criterial to agency by focusing on (i) self-awareness or consciousness and (ii) autonomy over own actions, distinguishing these from (iii) mere instincts or mechanistic reactions. As predicted, there was a reliable main effect for culture, $F(1,18)=10.83, p<.01, \eta 2$ $=.38$, with US participants providing substantially more content associated with consciousness (versus instincts) $(M=1.31 \%, S D=.92 \%)$ than Ngöbe participants $(M=0.24 \% S D=0.47 \%)$.

Following from a focus on relational agency, Ngöbe explanations were predicted to treat directedness as criterial to agency by focusing on (i) goal-directed needs or desires, (ii) teleological processes (e.g., "plants have the thought to grow"), and (iii) directed change or organized continuity of the entity's own states (e.g., growth, transformation, homeostasis or modulation). As expected, there was a reliable main effect for culture on directedness, $F(1,18)=24.97, p<.01, \eta 2=.58$, with Ngöbe participants providing much more such content $(M=4.32 \%, S D=1.92 \%)$ than US participants $(M=0.93 \%, S D=$ $0.96 \%)$.

\subsection{Interim summary}

The coding analysis revealed distinct cultural frameworks for agency as indicated by the different sets of framings, indicators, and criteria considered relevant for agency attribution, with the exception of internal indicators of agency. US respondents were more likely to frame agency in terms of a hierarchical scale where nonhuman entities are compared against human minds. They were also more likely to treat consciousness as a criterion for agency attribution. These findings are consistent with a concept of agency as a psychological capacity tied to having a mind (as humans know it). Anthropocentric framings were significantly less pronounced in Ngöbe explanations, which instead framed agency in terms of relational capacities expressed by many nonhuman kinds. Ngöbe also relied more on interactive indicators and behavioral directedness as criteria for agency. This is congruent with a concept of agency as a relational capacity based on an ecocentric prototype that encompasses multiple natural actors. On this model, agency may be conceptualized as a capacity with many unique endpoints more akin to a heterarchical network than a hierarchical progression along the scalae naturae (for related observations, see Hall, 2011; Narby, 2006).

A few examples illuminate how these observed cultural differences in explanatory frameworks impact the scope and nature of agency attribution. Consider whether a dog is capable of communication. Many Ngöbe respondents saw the act of barking as sufficient evidence for the affirmative, while US participants often sought evidence of conscious intentions before making a judgment (e.g., does the dog 
intend to communicate a message?). Additionally, US college justifications often implied that humans serve as arbiters of nonhuman agency (e.g., animals can't communicate "because they're difficult to interpret"), reflecting a view of humans as privileged knowers. When Ngöbe informants referred to humans' role in perceiving agency, they unanimously did so to affirm nonhuman agency ("The stones can tell you how long a life or time they were there"), and sometimes to affirm multiple forms of intelligent agency beyond the human (natural kinds "have their own form of communication, even though you don't know").

\subsection{Experiment 1: Discussion}

Experiment 1 revealed systematic cultural variation in agency attribution. We found that Ngöbe were more likely to attribute agency to plants and abiotic kinds than were US participants, who tended to restrict agency attributions to humans and animals with moderate extensions to complex artifacts, which they ranked reliably higher than did Ngöbe participants. The fact that the two groups did not differ on artifact inclusion rules out a generalized tendency for Ngöbe to attribute more agency to everything, which undermines the idea that Ngöbe agency attributions reflect a generalized metaphorical stance or related response bias. However, we did expect that US participants would be more likely than Ngöbe to include complex artifacts in the agency domain, which we did not find in this modest sample size. Still, each culture's agency attribution profile for natural versus artifact non-animal kinds, although not always statistically significant, was suggestive of distinct models. Converging evidence came from the coding analysis that revealed divergent explanatory frameworks for agency. US college participants tended to describe agency as a scalar, prototypically human capacity requiring consciousness, whereas Ngöbe adult participants tended to describe agency as a relational capacity expressed in the interactions and directed behaviors of many natural kinds. Ngöbe informants are familiar with complex artifacts such as cell phones and computers, but they were less likely to attribute agency to complex artifacts than to natural kinds, providing further evidence for an ecological grounds for inferring agency.

Taken together, these results are consistent with the proposal that Ngöbe adults hold an ecocentric model of agency as a relational capacity, and US college participants hold an anthropocentric model of agency as a psychological capacity. However, these findings are based on small samples and a limited number of capacity probes. An additional concern is that explicit verbal reports do not always align with underlying conceptual commitments, as other cultural research has shown (Astuti, 2001; Astuti et al., 2004). Stronger evidence for distinct cultural models would come from experimental manipulation of hypothesized agency frameworks, as well as a larger set of probes that can illuminate conceptual dimensions underlying agency frameworks across cultures. These were the objectives in Experiment 2.

\section{Experiment 2}

Experiment 2 sought to extend findings from Experiment 1 while providing additional insight into cultural concepts of agency. The first objective was to replicate the findings from Experiment 1 with larger samples (Ngöbe $n=24$, US $n=35$ ). We expected cultural differences in both inclusions and rankings for the three non-animal kinds (plants, abiotic kinds, and complex artifacts).

Second, we introduced a new experimental condition that framed agency as a relational capacity. In the original mind perception survey, agency predicates are presented with no grammatical object (e.g., Is $x$ capable of feeling hunger?), which implicitly frames the capacity as a property of the individual entity (we refer to this as the original condition). The Ngöbe explanations in Experiment 1 suggested that a more culturally fluent way of reasoning about agency capacities is to include the object(s) (e.g., Is $x$ capable of feeling hunger for food or nutrients?). Accordingly, in the relational condition we presented each agency predicate with grammatical objects that place the subject in relation to other entities, implicitly framing the capacity as a relational property. We predicted that Ngöbe would attribute greater agency to non-animal kinds (especially plants and abiotic kinds) in the relational condition as compared 
to the original condition, because a relational framing resonates with folkcommunication and facilitates an ecocentric perspective on agency. By contrast, we predicted that US participants would show no effect of condition (or possibly the opposite trend). ${ }^{8}$

Third, we selected a set of probes from the mind perception survey to assess the two-dimensional structure of mind perception, with three probes each for the dimensions we refer to as experience and cognition (Gray et al., 2007). ${ }^{9}$ Examining these dimensions of mind perception across cultures can offer insight into principles for inferring agency. Some researchers have argued that having internal representations is especially important for inferring capacities associated with the cognitive dimension, whereas having a body or biological functions is relevant for inferring experience (Knobe, 2011). If so, then this distinction can be leveraged to assess the inferential principles underlying agency frameworks. If the distinction between representational versus non-representational states is important to folkpsychology, then US participants should distinguish between cognition and experience in their attributions to non-animal kinds. If agency is inferred on the basis of relational rather than representational grounds, then for Ngöbe this distinction should be less pronounced. Additionally, we introduced two new probes associated with animacy, life and movement, to explore relationships between agency and animacy concepts. We predicted that they would correspond closely for Ngöbe participants because both are premised on a common capacity to interact, whereas agency and animacy should track separately among US participants who perceive them as distinct domains (folkpsychology and folkbiology).

One additional concern from Experiment 1 is that cultural differences may be due to language, given that Ngöbe interviewed in Spanish and US participants in English. To address potential language effects in Experiment 2, US bilingual Spanish-English speakers completed the task in Spanish using identical protocol to that used in Panama. We expected to observe no effects of language.

Other methodological changes in Experiment 2 allowed us address minor questions from Experiment 1 . We included a simple artifact (tote bag) to assess notions about non-computerized artifact capacities, on the prediction that agency attributions to this simple artifact would be uniformly low across cultures. This can provide a baseline measure of non-agency and also help rule out a positive response bias (as would be predicted by metaphor or related overextension accounts) in either sample. Additionally, Experiment 2 stimuli included two non-mammal animals (bird, frog) in addition to two of the mammals from Experiment 1 (dog, chimp) to assess whether cultural concepts of agency differ for distinct kinds of animals. We predicted that both Ngöbe and US individuals would ascribe agency to all animals, with similar patterns of ranking for mammals (generally higher) and non-mammals (generally lower).

\footnotetext{
${ }^{8}$ On folkpsychology, a mental-state term presumably points back to the individual entity regardless of the framing: feeling hunger, and feeling hunger for food, are both contingent on the subject having a mind to process feelings. Hence our prediction that the relational framing would have no effect on US attributions. Alternatively, a stronger hypothesis could be that US participants will be less likely to attribute agency to (perceived) insentient kinds in the relational condition, because the inclusion of a grammatical object stresses the explicit contents of a mental state (i.e., its "aboutness"), inducing a representational stance on agency and thereby setting a higher standard for capacity attribution than an open-ended predicate. For example, basic experiential capacities (fear) might shift to become interpreted as cognitive capacities (fear of enemies as a mental state with specific contents). Consistent with this possibility, we found that the relational condition decreased US agency attributions to plants for 4 of 6 capacities, but these trends did not reach reliability.

${ }^{9}$ Gray et al (2007) refer to the two dimensions of mind perception as experience and agency. They define agency specifically in terms of moral agency and responsibility, as distinct from the broader definition of agency we use here (namely, any entity that is perceived as capable of acting). For clarity, we refer to this dimension as cognition to distinguish it from our composite measure of agency (which includes all six capacities).
} 


\subsection{Participants}

Participants were Indigenous Ngöbe adults from the primary research community ${ }^{10}(n=24 ; 10$ females) and US college students ( $n=35 ; 18$ females). ${ }^{11}$ Ngöbe participants' ages ranged from 19-62 years $(M=37.21, S D=13.93)$. Experience with formal schooling ranged from $0-12$ years $(M=4.54, S D=3.24)$. Older informants tended to have less formal schooling than younger informants $(r=-.57, p<.01)$.

US participants' ages ranged from $18-23$ years $(M=19.00, S D=1.26)$. The US students came from the same university's introductory psychology class with similar demographics reported for Experiment 1. Recruitment, community permission, informed consent, and participant compensations for each sample were conducted in the same manner as in Experiment 1.

Participants also reported their occupation, religiosity (church attendance and three belief items used in Gray et al. 2007), language identity (their first language, and language spoken with their family), and experience with nature (farming/gardening, hunting/fishing, and pet experience, and urban/rural residence). (For item details see Appendix B1; for individual differences results, see Section 5.4.)

Condition. Participants were randomly assigned to an experimental condition, with approximately half of each group assigned to the original (Ngöbe: $n=12$; US: $n=19$ ) and relational (Ngöbe: $n=12$; US: $n=16$ ) conditions.

Language. The US college sample included 11 bilingual English-Spanish speakers, all of whom completed the study in Spanish. Language could not be randomly assigned due to the small number of bilingual participants. Among the Ngöbe sample, all participants completed the task in Spanish (occasionally, Ngöbere was used for clarification purposes).

\subsection{Methods}

\subsubsection{Materials and protocol}

Stimuli were color photographs of 14 entities depicted on cards (4" by $\left.4.25^{\prime \prime}\right)$, presented in random order. Entities were the same as in Experiment 1 (adult woman, infant, chimp, dog, two local plants, sun, ocean, rocks, robot, computer), with the addition of a bird, frog, and a tote bag (see Appendix B1). Eight capacity probes assessed three dimensions of agency: 1, cognition (communicate, memory, and morality); 2, experience (fear, pain, and hunger); and 3, animacy (alive and move). The first six probes are from the original mind perception survey. All capacity probes were presented to all participants. Order of presentation for the animacy probes was fixed, with move always presented first as a warm-up task, and alive always presented last. Intervening probes for the six agency capacities were presented in one of two counterbalanced orders (see Appendix B1). Each experimental condition used one of two question formats (relational or original) that applied to all six mind perception probes but not the animacy probes (see Appendix B2 for bilingual protocol).

Participants were asked to explain a subset of inclusions and exclusions on some of their capacity rankings. This was done to replicate the methods from Experiment 1, but explanations were not analyzed as part of the current experiment.

This experiment was administered as either the first or second task in a larger three-task interview (identical in the US and Panama). There were no effects of task order on agency attributions. As in Experiment 1, participants were interviewed in Spanish (Panama and US) or English (US) and

\footnotetext{
${ }^{10}$ Ngöbe participants in Experiment 2 were different individuals from those in Experiment 1, with the exception of 3 participants who participated in both studies. The two experiments were conducted far enough apart that task transfers were unlikely (Experiment 1: December 2011; Experiment 2: August-September, 2014).

${ }^{11}$ One additional Ngöbe participant and 14 additional US participants did not complete all probes and were not included in results reporting. The greater number of incomplete tasks for US participants was due to a time constraint during one academic quarter of data collection that truncated interviews.
} 
interviews were audio-recorded. Also as before, the Spanish protocols were forward- and back-translated and checked for local validity by S.G.G. in Panama.

\subsubsection{Data analysis}

We analyzed weighted ranks as in Experiment 1, with exclusions tied for last place (range: 1 - 14). Occasionally, participants ( $n=8$, all Ngöbe) reported that they did not know how to respond for a particular item. These items were treated as missing data. Most Ngöbe participants (6 of 8) who gave a "don't know" response did so only once for a single item on a single capacity ranking. The two Ngöbe participants who accounted for the majority (19/25) of "don't know" responses were excluded from rank results due to substantial missing data (see Appendix B3). ${ }^{12}$

\subsection{Results}

Our hypotheses predict the following for agency attributions to non-animal kinds (plants, abiotic kinds, and complex artifacts): (1) Ngöbe and US participants will attribute agency differently to these three kinds on a NKM versus AKM respectively; (2) the relational framing will increase Ngöbe but not US agency attributions; and (3) the relevant dimensions of agency attribution will differ, with US college participants distinguishing between the two mind perception dimensions more so than Ngöbe adults, and Ngöbe attributing agency in accordance with a third dimension of animacy more so than US participants. Finally, we conducted cultural consensus modeling to verify and extend the underlying conceptual models of agency implied by these results.

In testing these hypotheses we report results based on rates of inclusion, while reporting mean ranks for cultural consensus modeling in the next section. Reporting both sets of outcome measures would be redundant and inclusion rates provide the more straightforward account for hypothesis testing using standard comparative tests. ${ }^{13}$ Unless otherwise noted, statistical tests were conducted with a 2 (culture) by 2 (condition: original, relational) repeated-measures MANOVA with dimension (2: cognition, experience) and kind (6: humans, animals, plants, abiotic kinds, complex artifacts, simple artifact) as within-subjects variables. ${ }^{14}$ Where appropriate, degrees of freedom are adjusted using Huynh-Feldt estimates due to violation of assumption of sphericity (for kind $\left(\chi^{2}(14)=64.91, p<.001, \varepsilon=0.53\right.$; and dimension by kind $\left(\chi^{2}(14)=34.48, p<.01, \varepsilon=0.7\right)$. Where equality of means is violated, t-tests are reported with equal variances not assumed.

\subsubsection{Preliminary results}

Language. Among US participants, there were no reliable effects of Spanish versus English on rates of inclusion or mean rankings for any key outcome measure (i.e., overall agency attributions, attribution by kind or experimental condition, or the separate dimensions of cognition, experience, and animacy). Thus, we collapsed results across all US participants.

\footnotetext{
${ }^{12}$ It is safe to presume that a typical Ngöbe participant will have had less experience with standard interview techniques than a typical US college student. This may explain the greater frequency of "don't know" responses among Ngöbe as compared to US participants. Nonetheless, the majority of Ngöbe participants completed all probes or gave only a single "don't know" response. This fact should alleviate concerns regarding serious forms of cultural bias in our interview methods.

${ }^{13}$ All analyses reported below were also conducted for mean ranks and the story was roundly consistent with inclusion data; thus, results from rank data are presented only where they supplement the main findings from inclusion data.

${ }^{14}$ In analyses that include mind perception dimensions as a factor, some Ngöbe participants $(n=2$ for ranks; $n=1$ for inclusion) are excluded due to missing ("don't know") data on one dimension.
} 
Table 5. Mean percent inclusions for agency ${ }^{\mathrm{a}}$

\begin{tabular}{|c|c|c|c|c|c|c|c|}
\hline \multirow[b]{3}{*}{ Kind $^{c}$} & \multicolumn{7}{|c|}{ Mean percent inclusions } \\
\hline & \multicolumn{2}{|c|}{$\begin{array}{l}\text { Ngöbe } \\
(n=24)\end{array}$} & \multicolumn{2}{|c|}{$\begin{array}{c}\text { US } \\
(n=35)\end{array}$} & \multirow[b]{2}{*}{$t$-test ${ }^{b}$} & \multicolumn{2}{|c|}{$\begin{array}{l}\text { Overall } \\
(N=59)\end{array}$} \\
\hline & $M$ & $S D$ & $M$ & $S D$ & & $M$ & $S D$ \\
\hline Humans & $0.95 \mathrm{a}$ & $(0.06)$ & $0.99 \mathrm{a}$ & $(0.04)$ & $-2.71^{*}$ & $0.97 \mathrm{a}$ & $(0.05)$ \\
\hline Animals & $0.98_{\mathrm{a}}$ & $(0.04)$ & $0.98_{\mathrm{a}}$ & $(0.04)$ & $n s$ & $0.98_{a}$ & $(0.04)$ \\
\hline Plants & $0.70_{\mathrm{b}}$ & $(0.30)$ & $0.39_{b}$ & $(0.22)$ & $4.59^{* * *}$ & $0.52_{b}$ & $(0.30)$ \\
\hline Abiotic kinds & $0.31_{c}$ & $(0.30)$ & $0.04_{\mathrm{d}}$ & $(0.06)$ & $4.49^{* * *}$ & $0.15_{\mathrm{c}}$ & $(0.23)$ \\
\hline Complex artifacts & $0.15_{c, d}$ & $(0.20)$ & $0.24_{\mathrm{c}}$ & $(0.13)$ & $-1.86^{*}$ & $0.20_{\mathrm{c}}$ & $(0.17)$ \\
\hline Simple artifacts & $0.07_{d}$ & $(0.17)$ & $0.00_{\mathrm{e}}$ & $(0.03)$ & $n s$ & $0.03_{\mathrm{d}}$ & $(0.11)$ \\
\hline \multicolumn{8}{|c|}{ a Agency is a composite measure of memory, communication, morality, hunger, pain, and fear } \\
\hline \multicolumn{8}{|c|}{$\begin{array}{l}{ }^{\mathrm{c}} \text { Main effect for kind: Means in the same column not sharing the same subscript differ } \\
\text { significantly at } p<.05 \text { in pairwise tests (Bonferonni-adjusted). }\end{array}$} \\
\hline Note: ${ }^{*}$ Indicates $p<.0$ & & ${ }^{*} p<$ & & & & & \\
\hline
\end{tabular}

Mammals versus non-mammals. Patterns of agency attribution to mammals and non-mammals did not reliably differ across cultures. Thus, we collapsed all four items into a single category of animals. (For additional preliminary analyses, see Appendix B4).

\subsubsection{Cultural models of agency}

We begin by considering cultural variability in overall agency attributions across all six capacities. As hypothesized, we found a reliable interaction of culture by kind, $F(3.07,165.73)=33.1, p<$ $.001, \eta^{2}=0.38 .{ }^{15}$ Follow-up comparisons showed that, as predicted on an ecocentric prototype, Ngöbe were more likely than US participants to include non-animal natural kinds (plants and abiotic kinds) in their agency attributions (Plants: $t(57)=4.59, p<.001, d=1.24$; Abiotic kinds: $t(24.29)=4.49, p<.001, d=$ 1.44) (see Table 5). Corresponding to an anthropocentric prototype, US individuals were more likely than Ngöbe to include complex artifacts in agency attributions, $t(57)=-2.00, p=.05, d=-0.54$.

Within each culture, the profile of agency attribution for non-animal kinds also differed (see Table 5). Ngöbe followed the predicted NKM privileging plants and abiotic kinds above complex artifacts, as evidenced by inclusion rates and ranks (where plants and abiotic kinds are both ranked significantly higher than complex artifacts, see Appendix Table B1). Plants were included at rates well above chance, $t(23)=3.30, p<.01$, suggesting that the agency domain for Ngöbe includes plants as well as animals and humans. US attributions followed a partial AKM with complex artifacts accorded greater agency than abiotic kinds but not plants, and this pattern was mirrored in ranks (where complex artifacts were ranked above abiotic kinds but not reliably different from plants, see Table B1). Among US college participants, plant inclusion is significantly below chance, $t(34)=-2.93, p<.01$, indicating that plants stand farther outside the agency domain on this model.

As expected, the two cultural groups did not differ in inclusion rates for animals, $t(57)=-0.44, p=$ $.66, d=-0.12$. US college participants were reliably more likely than Ngöbe adults to include humans, $t(35.5)=-2.49, p<.05, d=-0.73$, but this difference was of slight magnitude (mean difference $=.04$ ) and both groups were near ceiling in their attributions.

\subsubsection{Experimental condition: Relational framings of agency}


Ngöbe agency attributions differed more across experimental conditions than did US attributions, as indicated by the reliable 3-way interaction for kind, culture, and condition $(F(3.07,165.73)$ $=5.91, p<.01, \eta^{2}=0.10$ ). To further investigate these effects, we analyzed agency attributions by condition separately for each cultural group. Inclusions were analyzed with a 2-factor (condition: original, relational) repeated-measures ANOVA with kind (6) as a within-subjects variable. We continue to focus on agency as a composite variable of all six capacities, with accompanying tests of each capacity to clarify key findings. ${ }^{16}$ We consider Ngöbe and US results in turn, which are summarized in Figure 1 (see Appendix B5 for detailed results).

Ngöbe adults. As expected, Ngöbe rates of agency attribution were higher in the relational condition $(M=.60, S D=.15)$ than the original condition $(M=.46, S D=.07), F(1,22)=8.78, p<.01, \eta^{2}=0.29$, and this interacted with kind, $F(2.54,55.83)=3.77, p<.05, \eta^{2}=0.15$. Follow-up tests confirmed that Ngöbe were reliably more likely to include abiotic kinds, complex artifacts, and simple artifacts in the relational than original condition $(p s<$ or $=.05)$. The same trend held for plants but did not reach significance $(p=$ .14)

Separate analyses of Ngöbe inclusions for each capacity confirmed that the effect of condition held for four of six capacities ( $p s<.05$ ), with the two exceptions being morality and pain, where the same trends held but did not reach significance. This systematic pattern is consistent with the hypothesis that these capacities recruit a common conceptual framework for agency.

US college students. US agency attributions were unaffected by experimental condition, as indicated by the non-significant interaction of condition by kind, $F(2.22,73.25)=1.58, p=.21, \eta^{2}=0.05$. There were no reliable differences in agency attribution across conditions or mind perception dimensions for any kind.

Cross-cultural comparisons. As noted above, the significant 3-way interaction of kind, culture, and condition indicated that the effect of condition varied across cultures. We analyzed cross-cultural differences for each kind across the two conditions, confirming that key differences held reliably for each

Figure 1: Inclusion in agency attributions for each kind by condition and culture

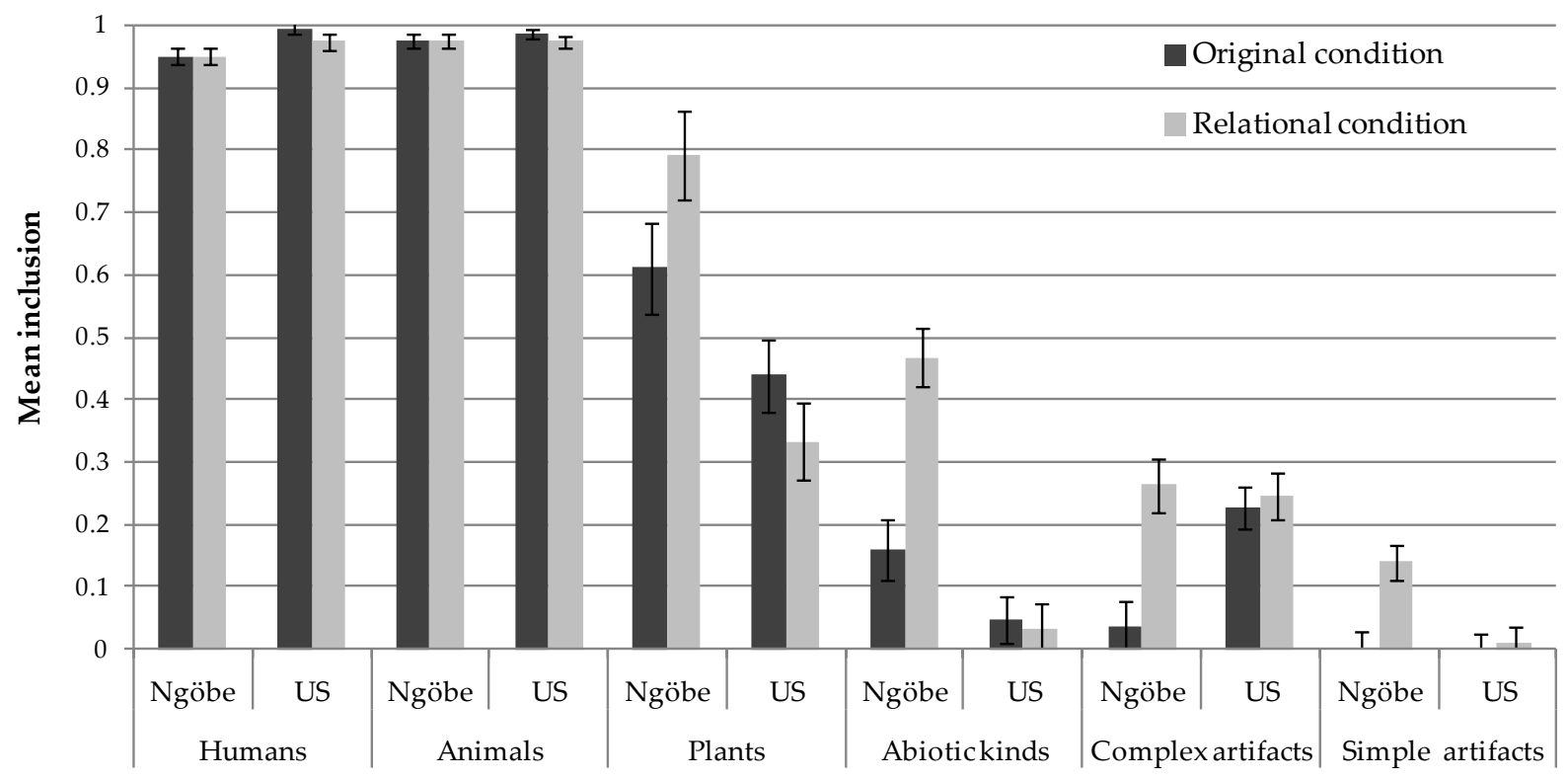

Kind

${ }^{16}$ The effect of condition did not vary across the two mind perception dimensions, so these were collapsed for an overall analysis of agency. 
non-animal kind across both conditions, with the sole exception of complex artifacts in the relational condition (where the two groups did not differ). ${ }^{17}$ The finding that cultural differences hold in both conditions strengthens the interpretation of two distinct cultural models, insofar as a given framing does not appear to push one group away from an underlying "default" model that is actually shared with the other cultural group.

\subsubsection{Conceptual dimensions}

Next we consider whether cultural agency concepts track differently along the mind perception dimensions of experience and cognition, and how these relate to animacy concepts. So far we have treated agency as a unified construct, but there may be important distinctions within this domain. As noted, prior research among Western samples has shown that folkpsychological attributions track two separate dimensions corresponding to faculties perceived as either experiential (sensory) or cognitive (representational) (Gray, et al., 2007). For example, human-made artifacts such as robots are seen as high on cognition but low on experiential capacities, whereas human infants are seen as high on experience but low on cognition. If representational capacities are a salient principle for inferring agency on folkpsychology but not folkcommunication, then we should expect agency attribution patterns for nonanimal kinds to differ on these two dimensions for US college participants, but not for Ngöbe adult participants. The inclusion results reported above provide suggestive evidence for this possibility, as US college participants included plants at rates below chance but comparable to complex artifacts. This may reflect distinct notions of what plants "do" (i.e., have biological states) and what computers "do" (i.e., have representational states).

For Ngöbe, we predicted that a third dimension of animacy would be relevant to agency attributions. We have thus far contrasted abiotic kinds (sun, ocean, rocks) with biological kinds (plants, animals, and humans) as if only the latter are alive, following standard Western notions. But the concept of animacy, operationalized here in terms of the capacity for life and movement, may tap into different conceptual frameworks across cultures. On folkcommunication, we expected Ngöbe attributions of both cognition and experience to correspond closely with animacy judgments because all three dimensions may be inferred on the basis of a capacity to relate and interact with the environment.

\footnotetext{
${ }^{17}$ In both the original and relational conditions, Ngöbe adults were significantly more likely than US college participant to include plants and abiotic kinds, $p s<.001$. In the original condition only, US participants were more likely than Ngöbe to include complex artifacts, $p<.001$. Simple artifacts did not reliably differ across cultures in either condition.
} 
Table 7. Mean percent inclusions for cognition and experience ${ }^{a}$

\begin{tabular}{|c|c|c|c|c|c|c|c|c|c|c|}
\hline \multirow[b]{3}{*}{ Kind } & \multicolumn{5}{|c|}{ Cognition } & \multicolumn{5}{|c|}{ Experience } \\
\hline & \multicolumn{2}{|c|}{$\begin{array}{l}\text { Ngöbe } \\
(n=24)\end{array}$} & \multicolumn{2}{|c|}{$\begin{array}{c}\text { US } \\
(n=35)\end{array}$} & \multirow[b]{2}{*}{$t$-test ${ }^{\mathrm{b}}$} & \multicolumn{2}{|c|}{$\begin{array}{l}\text { Ngöbe } \\
(n=24)\end{array}$} & \multicolumn{2}{|c|}{$\begin{array}{c}\text { US } \\
(n=35)\end{array}$} & \multirow[b]{2}{*}{$t$-test } \\
\hline & $M$ & $S D$ & $M$ & $S D$ & & $M$ & $S D$ & $M$ & $S D$ & \\
\hline Humans & $0.91_{a}$ & $(0.11)$ & $0.97 a$ & $(0.08)$ & $-2.4^{*}$ & $0.99 \mathrm{a}$ & $(0.03)$ & $1.00_{a}$ & $(0.00)$ & $n s$ \\
\hline Animals & $0.95_{a}$ & $(0.08)$ & $0.96_{a}$ & $(0.08)$ & ns & $1.00_{\mathrm{a}}$ & $(0.00)$ & $1.00_{a}$ & $(0.00)$ & -- \\
\hline Plants & $0.59_{b}$ & $(0.38)$ & $0.35_{b}$ & $(0.31)$ & $2.62^{*}$ & $0.85_{b}$ & $(0.22)$ & $0.43_{b}$ & $(0.30)$ & $5.02^{* * *}$ \\
\hline Abiotic kinds & $0.36_{\mathrm{c}}$ & $(0.33)$ & $0.07_{\mathrm{c}}$ & $(0.12)$ & $4.09^{* * *}$ & $0.28_{\mathrm{c}}$ & $(0.30)$ & $0.01_{\mathrm{c}}$ & $(0.04)$ & $4.43^{* * *}$ \\
\hline Complex artifacts & $0.17_{c, d}$ & $(0.23)$ & $0.40_{\mathrm{b}}$ & $(0.26)$ & $-3.36^{* *}$ & $0.12_{d}$ & $(0.21)$ & $0.08_{c}$ & $(0.15)$ & $n s$ \\
\hline Simple artifacts & $0.06_{\mathrm{d}}$ & $(0.16)$ & $0.01_{d}$ & $(0.06)$ & $n s$ & $0.09_{d}$ & $(0.21)$ & $0.00_{\mathrm{c}}$ & $(0.00)$ & ns \\
\hline
\end{tabular}

${ }^{a}$ Cognition is a composite of memory, communication, and morality. Experience is a composite of hunger, pain, and fear.

${ }^{\mathrm{b}} \mathrm{T}$-tests compare mean inclusion rates across cultures for the kind in that row.

${ }^{\mathrm{c}}$ Means in the same column not sharing the same subscript differ at $p<.05$ in pairwise tests (Bonferonni-adjusted).

Note: * Indicates $p<.05 ;{ }^{* *} p<.01 ;{ }^{* *} p<.001$.

\subsubsection{Mind perception dimensions}

As summarized in Table 7, agency attributions differed across the two dimensions for both US and Ngöbe participants, but did so in distinct ways. To follow up the reliable 3-way interaction of kind, culture, and mind perception dimension, $F(2.79,150.5)=4.62, p<.01, \eta^{2}=0.08$, we explored rates of inclusion by dimension within each culture using repeated-measures ANOVAS with dimension (2: experience, cognition) and kind (6) as within-subjects variables. Where appropriate, degrees of freedom are adjusted using Huynh-Feldt estimates due to violation of assumption of sphericity for kind (US: $\chi^{2}(14)=147.75, p<.001, \varepsilon=0.42$; Ngöbe: $\chi^{2}(14)=78.62, p<.001, \varepsilon=0.53$ ) and kind by dimension (US: $\chi^{2}(14)=153.8, p<.001, \varepsilon=0.47$; Ngöbe: $\left.\chi^{2}(14)=51.73, p<.001, \varepsilon=0.62\right)$. Each mind perception dimension represented a composite variable of three capacities (experience: fear, hunger, pain; cognition: morality, communication, memory). As a complementary analysis, we also examined correlations between mean ranks (see Table 8) to assess the strength of relations among conceptual dimensions.

The key cultural differences in agency attribution to non-animal kinds held reliably across both experience and cognition, with the sole exception that complex artifacts were similarly low on experience for both groups $(p=.30)$ (see Table 7). Thus, we focus on how these dimensions differed within each culture for US and Ngöbe participants in turn.

US college students. As predicted, US agency attributions differed across the two mind perception dimensions, $F(1,34)=4.47, p<.05, \eta^{2}=0.12$, and this varied by kind $F(2.35,79.93)=15.36, p<.001, \eta^{2}=$ 0.31 . Prior literature suggests that complex artifacts should be seen as having more cognitive capacities relative to experiential capacities, on the basis that computers can represent information but are not alive (Knobe, 2011). The opposite should hold for plants because they are considered alive but lacking in representational faculties. Consistent with this, complex artifacts were more likely to be included in cognition than experience (complex artifacts: $t(34)=-5.65, p<.001, d=-1.51$ ), as were (surprisingly) abiotic kinds $(t(34)=-3.11, p<.01, d=-0.78)$. The opposite trend held for plants, which were included more often on experience than cognition, but this did not reach reliability, $p=.30 .18$

Correlations between US mean ranks for each kind further illuminated this two-dimensional structure (see Table 8). If experience and cognition represent distinct dimensions of agency for US

\footnotetext{
${ }^{18}$ Inclusion rates for humans and animals were also higher on experience than cognition (humans: $t(34)=2.24, p<.05$, $d=0.54$; animals: $t(34)=2.94, p<.01, d=0.71$ ), but these differences were small as attributions for both kinds were at or near ceiling.
} 
Table 8: Intercorrelations between mean ranks across dimensions by culture

\begin{tabular}{|c|c|c|c|c|}
\hline \multirow{4}{*}{ Kind } & \multicolumn{2}{|c|}{ US } & \multicolumn{2}{|c|}{ Ngöbe } \\
\hline & Experience $^{\mathrm{a}}$ - & Agency $^{\mathrm{a}}$ - & Experience $^{\mathrm{a}}$ - & Agency $^{\mathrm{a}}$ - \\
\hline & Cognition $^{\mathrm{a}}$ & Animacy $^{b}$ & Cognition $^{\mathrm{a}}$ & Animacy $^{b}$ \\
\hline & $r$ & $r$ & $r$ & $r$ \\
\hline Humans & $.43^{* *}$ & $.55^{* * *}$ & $.56^{* *}$ & $.42^{*}$ \\
\hline Animals & .05 & -.02 & $.65^{* * *}$ & $.76^{* * *}$ \\
\hline Plants & .28 & .13 & $.69^{* * *}$ & $.55^{* *}$ \\
\hline Abiotic kinds & .03 & $-.31^{\dagger}$ & $.56^{* *}$ & $.38^{+}$ \\
\hline Complex artifacts & .12 & $.44^{* *}$ & $.55^{* *}$ & $.44^{*}$ \\
\hline Simple artifacts & -.08 & $.66^{* * *}$ & $.57^{* *}$ & .22 \\
\hline
\end{tabular}

participants, then these two sets of attributions should be extended on the basis of distinctive considerations for each non-animal kind such that ranks are not reliably correlated across dimensions. Consistent with this, US experience and cognition ranks were not reliably correlated for any non-animal kind (all $r s=n s$ ). Instead, cognition and experience are reliably correlated only for humans, which would be expected if humans serve as the prototype for both dimensions.

Ngöbe adults. We expected that Ngöbe would not distinguish between the dimensions of experience and cognition, but Ngöbe inclusions did differ across the two dimensions, $F(1,22)=4.86, p<$ $.05, \eta^{2}=0.18$, and this varied by kind, $F(3.10,68.26)=10.26, p<.001, \eta^{2}=0.32$. Like US participants, Ngöbe more frequently included plants in experience than cognition, $t(23)=3.66, p<.01, d=0.68$, whereas abiotic kinds were more often included in cognition than experience, $t(22)=-2.2, p<.05, d=-0.31$. Unlike US college participants, however, there was no reliable difference for Ngöbe adult attributions to complex artifacts across the two dimensions, $t(23)=-1.37, p=.18, d=-0.23 .{ }^{19}$ The fact that abiotic kinds were high on cognition while complex artifacts were low indicates that some principle other than representational information processing must be central to the Ngöbe framework for inferring this dimension of mind perception.

Although Ngöbe agency attributions did differ across dimensions, they were still closely related. Unlike the US college sample, Ngöbe ranks for experience and cognition were significantly correlated for every kind, both animal and non-animal ( $p s<.05$ ). This is consistent with our prediction that for Ngöbe informants, experience and cognition are inferred on the basis of similar principles.

In sum, US college participants differentiated more strongly than Ngöbe adult participants between cognition and experience for all three non-animal kinds. Both groups appeared to share a biology-based model for experience, but US attributions for cognition followed an artifact kinds model whereas Ngöbe followed a natural kinds model. The pronounced differences on cognition for abiotic kinds versus complex artifacts suggest that different inferential principles are at stake in these two models of agency. One relevant factor may be concepts of life, to which we now turn.

\footnotetext{
${ }^{19}$ As seen for US participants, Ngöbe attributions for experience versus cognition also differed for both humans and animals, but again this was a minor effect with all inclusions at or near ceiling (humans: $t(23)=3.72, p<.01, d=1.05$; animals: $t(23)=3.06, p<.01, d=0.90)$.
} 


\subsubsection{Animacy}

We propose that Ngöbe adults may be using a unified conceptual framework for agency that infers experience, cognition, and life on the basis of similar relational principles. If so, then their high attributions of cognition to abiotic kinds could reflect the possibility that Ngöbe individuals also see these natural kinds as animate.

Cross-cultural differences. Cultural differences in concepts of animacy for abiotic kinds (sun, ocean, rocks) were dramatic. Ngöbe were far more likely than US participants to recognize abiotic kinds as alive (Ngöbe: $M=.75 ; S D=.26$; US: $M=.11 ; S D=.23$ ), $t(57)=9.86, p<.001, d=2.66$, and capable of movement (Ngöbe: $M=.76 ; S D=.18$; US: $M=.54 ; S D=.33$ ), $t(54.79)=3.26, p<.01, d=0.79 .{ }^{20}$ The sun and ocean were together ranked above all other kinds on animate capacities by the majority of Ngöbe participants (11/22 for life, 15/22 for movement), in stark contrast to US college participants (1/35 for life, 5/35 for movement). The fact that the sun and ocean were ranked so prominently in Ngöbe models of animacy suggests their prototypical status as animate forces. In contrast, US life attributions followed a categorical biological kinds model: plants, animals, and humans were almost always included and all other kinds excluded. US movement attributions were more graded, with abiotic kinds and complex artifacts included at levels near $50 \%$.

Otherwise, the two groups converged on life and movement inclusions for each kind, with a minor difference for complex artifacts, $t(57)=-2.56, p=.05, d=-0.69$, such that US college participants were more likely than Ngöbe adults to say that robots (but not computers) could move (US: $M=.71, S D=.46$; Ngöbe: $M=.46, S D=.51$ ). This could reflect different levels of familiarity with robots, and/or different interpretations of movement as autonomous versus automated.

One other comparative property of life ranks should be noted. Among US college participants, all living kinds (plants, animals, humans) were often ranked on a single level and everything else excluded, representing a binary concept of alive/not alive. Interestingly, Ngöbe rankings for life were just as graded as for any other agency capacity. Evidently, entities may possess distinct degrees of life force on the Ngöbe folktheory of agency.

Animacy and agency. The proposal for folkcommunication would predict a strong relationship between animacy and agency for Ngöbe participants, insofar as both capacity dimensions are inferred from similar principles. As summarized in Table 8, Ngöbe attributions of animacy and agency were indeed reliably correlated for each kind $(p s<.05)$ except abiotic kinds, which only reached marginal significance $(p=.08)$. We did not expect a strong relationship for agency and animacy among US college attributions given that the two constructs should tap into distinct inferential principles for folkpsychology and folkbiology respectively. Indeed, the two constructs were reliably correlated only for humans but none of the natural nonhuman kinds. Interestingly, the two constructs were also correlated for human-made artifacts, but this is based on a small number of US college students who attributed animacy to those kinds.

${ }^{20}$ Examining life attributions for each of the three abiotic kinds shows that Ngöbe roundly agree on the living kind status of the ocean $(\mathrm{M}=.96, \mathrm{SD}=.20)$ and the sun $(\mathrm{M}=.88, \mathrm{SD}=.34)$, in stark contrast to the US majority conception of these kinds as lifeless (ocean: $\mathrm{M}=.14$, $\mathrm{SD}=.36$; sun: $\mathrm{M}=.20 ; \mathrm{SD}=.41)(p s<.001)$. Ngöbe were also significantly more likely to include the sun and ocean in movement attributions ( $p s<.05)$, although there was greater crosscultural agreement on this point (Ngöbe sun: $\mathrm{M}=.96, \mathrm{SD}=.20 ;$ Ngöbe ocean: $\mathrm{M}=1.00, \mathrm{SD}=.00 ; \mathrm{US}$ sun: $\mathrm{M}=.63, \mathrm{SD}=$ .49 ; US ocean: $\mathrm{M}=.74, \mathrm{SD}=.44$ ). Rocks figure less prominently in Ngöbe animacy attributions (inclusions for life: $\mathrm{M}=$ $.42, \mathrm{SD}=50$; and movement: $\mathrm{M}=.33$; $\mathrm{SD}=.48)$; but this still contrasted with US participants' total denial of life to rocks $(\mathrm{M}=0, \mathrm{SD}=0), p<.001$, although movement attributions were more similar across cultures $(\mathrm{US}$ : $\mathrm{M}=.26, \mathrm{SD}=$ $.44)(\mathrm{p}=.54)$. Notably, rocks still stand far above complex artifacts in Ngöbe attributions of life $(\mathrm{M}=.08, \mathrm{SD}=.28$, identical for robot and computer), which differed not at all from simple artifacts $(\mathrm{M}=.08, \mathrm{SD}=.28)$. Interestingly, US participants tended to attribute life to robots $(\mathrm{M}=.14, \mathrm{SD}=.36)$ and computers $(\mathrm{M}=.11, \mathrm{SD}=.32)$ more often than Ngöbe participants, but these differences were not reliable ( $p s=.50$ and .71 , respectively). 


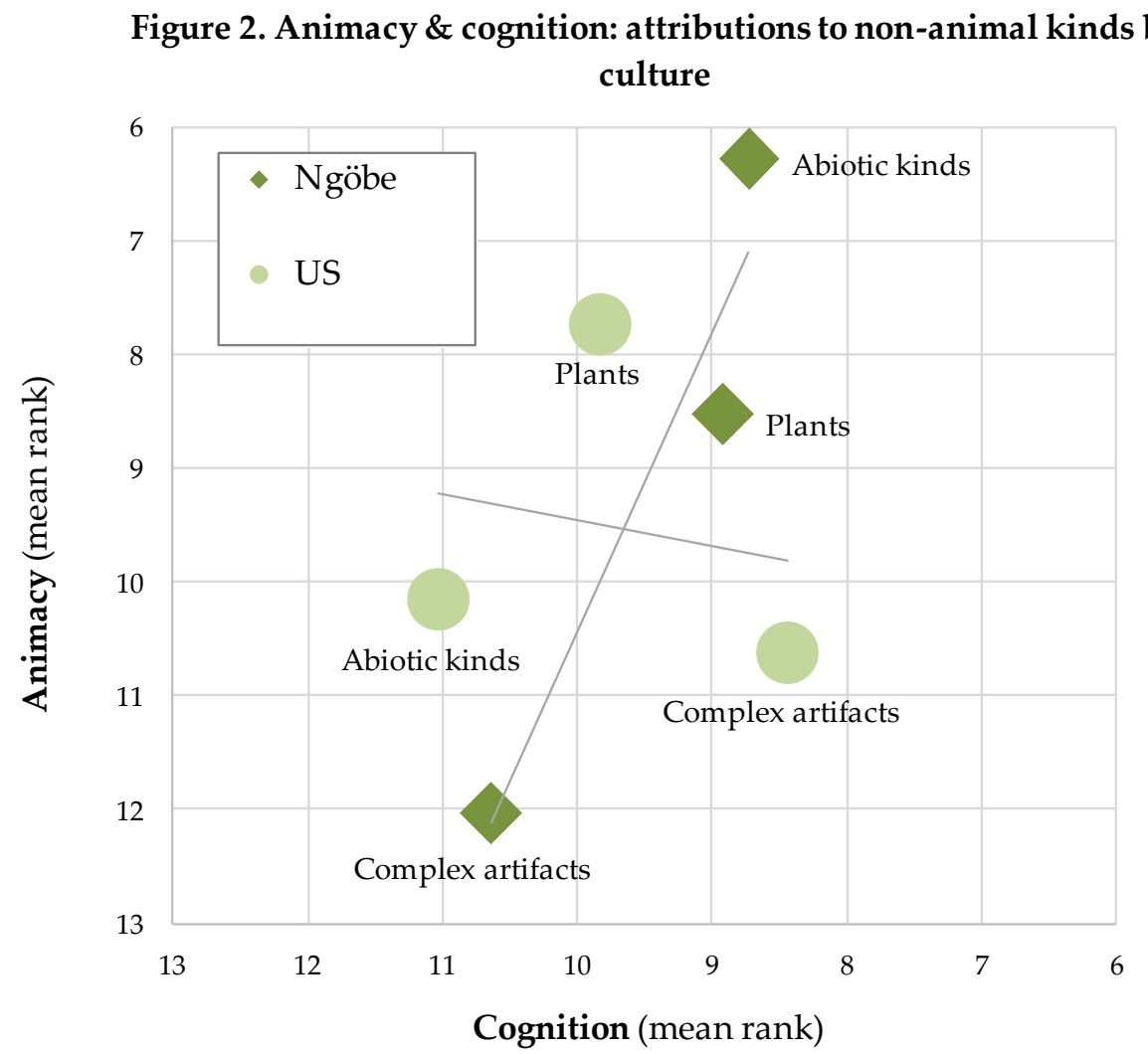

Animacy judgments are especially informative when examined in relation to cognition, where the largest cultural differences emerged with respect to non-animal kinds. It is clear from Figure 2 that Ngöbe attributions of animacy and cognition track together across all three non-animal kinds: the more alive an entity is perceived to be, the more it is seen as capable of cognition. In direct contrast, US attributions of cognition and animacy to non-animal kinds bore no relationship: life and cognition were inferred independently of one another.

These striking cultural differences in animacy concepts indicate that the mind perception dimension known as cognition may tap into very distinct conceptions across cultures. Our results suggest that the Ngöbe framework for "cognitive" agency may be grounded in a view of animacy as a graded force where powerful abiotic kinds like the sun or ocean are prototypes. If so, then this mind perception dimension might be more appropriately labeled as animacy than cognition. This contrasts with the US college student view of cognition as a representational information processing capacity, where inferences of cognitive capacity are evidently based on distinct principles from those used for inferring animacy.

\subsection{Detecting multiple conceptual models without "culture"}

\subsubsection{Cultural consensus modeling}

In this section our analysis shifts from asking what kind of conceptual model a culture has, to what kinds of cultures a model has. Setting aside any predefined cultural groupings, we used cultural consensus modeling to identify whether multiple latent conceptual models of agency are present in the dataset (collapsing across US and Ngöbe participants), and to explore between- and within-culture variations on these conceptual models. Our first objective was to determine if there are one or more consensus conceptual models. This is an important step beyond the cultural differences reported above because two groups can differ even while one group fails to converge on a consensus model (Le Guen et 
al., 2013). If more than one consensus is detected this will indicate that multiple coherent models give rise to the observed cultural differences. Second, we are interested in the agency attribution profile of each consensus model, including which items are the most difficult to reach consensus on. Comparing these rank profiles can shed light on the prototypes and forms of competence that structure each consensus model. Third, we are interested in how participants cluster into the consensus models according to their actual cultural membership and individual characteristics, as well as how individual differences may predict competence within each model. This will enrich understanding of across- and within-group variability and point to individual factors that may influence one's conceptual model of agency. Our modeling is conducted on the mean rank data to complement and extend the prior inclusion results. Cultural consensus modeling (CCM) (Romney et al., 1986) has been applied extensively in prior cultural research on biological cognition (Atran \& Medin, 2008; Medin et al., 2007). Conceptually similar to factor analysis, CCM determines if a single underlying model exists, as well as whether patterns of residual agreement beyond the overall consensus suggest additional models that hold for subgroups in the sample. Each participant is assigned a cultural "competence," where higher scores on a factor loading indicate that an individual's responses are closer to the cultural consensus. CCM also calculates the consensus model "answer key" with a competency weighted consensus rating for each item, providing a more reliable approximation of the common truth than traditional data aggregation techniques (France \& Batchelder 2014).

Recent advances in CCM provide a model-based way to derive multicultural consensus from continuous response data (Anders, et al., 2014). This new Bayesian inferential approach to consensus modeling using a modeling program called CCTpack (Anders, 2013) offers two key advantages over prior CCM techniques. 1. It treats culture as a latent variable by providing a model-based way to assess whether separate consensus models exist within a sample and to identify each participant's consensus group membership (rather than relying on known cultural membership specified in advance, as in traditional CCM methods). This confers a theoretical advantage over statistical models that treat culture as a fixed independent variable, by focusing on conceptual models and allowing cultural membership to remain latent. To emphasize this analytic distinction, we refer henceforth to "conceptual/consensus models" rather than "cultural models." 2. CCTpack introduces a new response precision parameter to assess whether some items are more difficult than others, unlike traditional CCM methods that assume all items are equally difficult. The resulting consensus model answer key accounts for variable item difficulty in addition to respondent competence, providing a more sensitive estimate of the shared conceptual model.

\subsubsection{Methods and procedure}

Using the CCTpack R software package (Anders, 2013), we applied the Bayesian CCT model to the agency ranks data (including both mind perception dimensions but not animacy) ${ }^{21}$ from all US and Ngöbe participants together $(N=57)$. The participant-level mean ranks for each kind on both experience and cognition were subjected to cultural consensus modeling at the item level $(\mathrm{k}=14)$, for a 57 (participants) X 28 (14 items $X 2$ dimensions) matrix. For ease of interpretation results are presented at the kind level.

In CCTpack, analysis is executed by running many iterations of a given model with various parameters adjusted between models (here, the number of derived "cultures" or consensuses and

\footnotetext{
${ }^{21} \mathrm{CCM}$ was conducted on the two mind perception dimensions excluding animacy for a more conservative test of latent conceptual models, insofar as the stark cultural difference in life judgments for abiotic kinds could contribute disproportionately to detection of consensus models and membership. Nonetheless, the consensus models for experience and cognition remain virtually identical when the CCM included Alive, and membership clustering results were similar (27 of 35 US participants into Culture 1 and 21 of 22 Ngöbe participants into Culture 2).
} 
difficulty of items). Two posterior predictive checks are used to compare which model best fits the actual data (for details, see Anders, 2013; Anders et al., 2014). 1. To check that the consensus structure of the data is appropriately fit by the model (i.e., the appropriate number of consensuses is identified), a scree plot of eigenvalues assesses the fit between the model-generated estimates and the actual structure of the data. 2. To check whether the item difficulty parameter should be treated as uniform (i.e., homogenous item difficulty) or variable (i.e., heterogeneous item difficulty), a variance dispersion index (VDI) reports how well the model captures differences across items due to response variability; these VDIs are compared and the model with better fit is selected.

Our use of CCTpack constitutes exploratory work on the capacity of the Continuous Response

801 Model (CRM) to handle multicultural ranking data, which has not been done before (see Appendix B6).

802 Our results contribute to the CCM literature by confirming the robustness of this model with rank data.

\subsubsection{Results}

\subsubsection{Model selection and identification of latent conceptual models}

The data were simulated using a series of models with 1, 2, and 3 consensuses, with and without heterogeneous item difficulty.

Item difficulty. The VDI check for item difficulty indicated that a model assuming heterogeneous (versus homogenous) item difficulty better fit the data.

Consensus fit. The scree plots of eigenvalues showed that the first factor was substantially larger than subsequent factors, with a second factor evident but less pronounced. Posterior predictive checks showed that the data structure was well fit by both a one- and two-consensus model, but a threeconsensus model failed to fit the data. ${ }^{22}$ This suggests an overarching consensus accompanied by two subgroups. The overall consensus was expected due to the substantial agreement on ranks for humans and animals, while a two-consensus model would be expected to pick up additional patterned variability concerning non-animal kinds. In the past, we have consistently found a good overall consensus paired with systematic subgroup differences (e.g., Medin, et al., 2007). Thus, we selected a two-consensus model with heterogeneous item difficulty.

818 5.4.3.2. Consensus Models

${ }^{22}$ A 3-consensus model was unable to reach convergence of modeling chains (Anders, 2014), indicating that there were no detectable third components and/or too many degrees of freedom. 
Figure 3: Model-based consensus models for agency by dimension

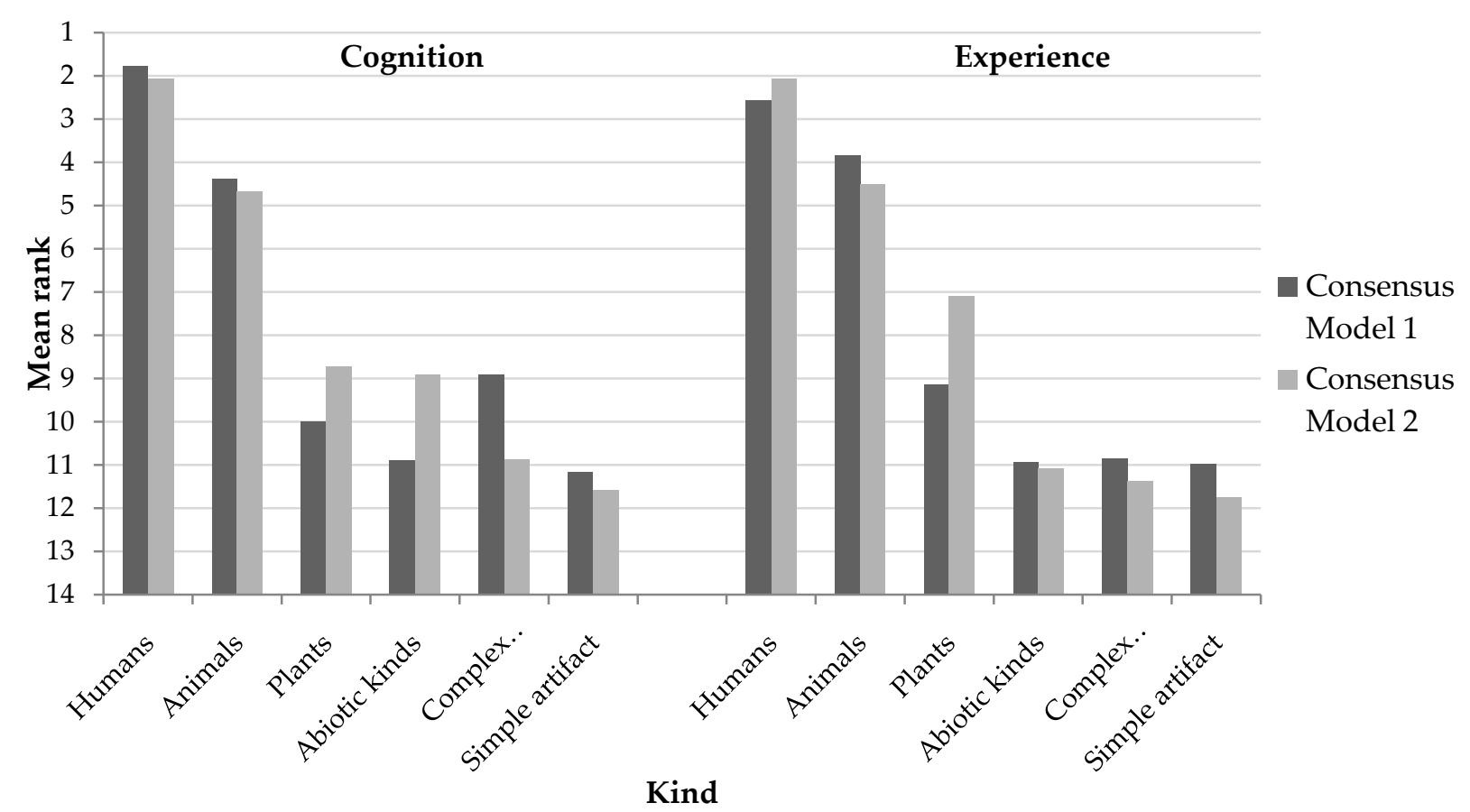

The model identified two consensuses latent in the agency rank responses (see Figure 3). The two consensus models that emerged were consistent with hypothesized frameworks for ecocentric versus anthropocentric agency. The items that are in largest disagreement between the two models are nonanimal kinds, reflecting that plants, abiotic kinds, and complex artifacts were organized differently across the two consensuses. The first consensus model for agency fit an artifact kinds model (AKM) for cognition (complex artifacts were ranked above both abiotic kinds and plants), but experience followed a biology-based natural kinds model (NKM) (plants ranked above both complex artifacts and abiotic kinds). The second consensus model followed a NKM for both cognition and experience, with cognition relatively ecology-based (abiotic kinds and plants ranked together above complex artifacts) and experience relatively biology-based (plants ranked higher than both abiotic kinds and complex artifacts). Comparing the two consensus models, cognition is where the more pronounced differences arise. Model 1 appears to treat cognition as a property of information-processing artifacts (only), whereas Model 2 treats cognition as a property of all natural kinds but not artifacts.

Item difficulty. We used item difficulty results to illuminate the forms of competence associated with each conceptual model. One unique strength of CCM is that it goes beyond sample means to assess each individual's level of competence on the consensus model. CCTpack improves on this feature by analyzing item difficulty for each item in the model. The most difficult items are the hardest to reach consensus on, and so can be used to distinguish more from less competent members of a consensus group. ${ }^{23}$

On Model 1, complex artifacts and humans were the most difficult item kinds for cognition, such that greater competence was correlated with higher rankings for complex artifacts $(r=-.35, p<.05)$ and lower rankings for humans $(r=.34, p<.05)$, indicating that these two kinds shifted places near the top of

\footnotetext{
${ }^{23}$ This interpretation of item difficulty builds on the property of the CCM model that in general, more competent respondents will tend to get the hard items correct more frequently than less competent members.
} 
rank hierarchies depending on competence. ${ }^{24}$ For experience, humans and animals were the most difficult items, such that those with greater competence ranked animals lower $(r=.38, p<.05)$ and tended to rank humans higher $(r=-.28, p=.08) .{ }^{25}$ (Recall that the human stimuli included an infant, which accounts for variations in mean rank of humans.) On Model 2, abiotic kinds and humans were the most difficult item kinds for both cognition and experience. Greater competence was correlated with ranking abiotic kinds higher $(r s=-.90$ and -.67 for experience and cognition respectively, $p s<.001)$ and humans lower $(r s=.53$ and $.49, p s<.05)$, indicating that these two kinds shifted places near the top of rank hierarchies depending on competence.

These results suggest that the two consensus models represent qualitatively distinct forms of reasoning about agency. For the anthropocentrically oriented Model 1, greater competence means an increased recognition of complex artifacts' capacity for cognition, coupled with lower estimation of animals' capacity for experience. For the ecocentrically oriented Model 2, greater competence means greater appreciation of both the cognitive and experiential capacities of abiotic kinds like the sun and ocean.

\subsubsection{Consensus model membership}

These item-specific differences in competence motivate further questions concerning individual and conceptual variability in consensus model membership, both across and within the two models.

Consensus group membership. The model detected and clustered individual participants into the two consensus models in close correspondence with actual cultural membership. The majority of US college participants (31 of 35) were clustered with Model 1, and the majority of Ngöbe (14 of 22) with Model 2. It is notable that US college participants clustered so strongly into a single model despite being the larger sample and being drawn from two distinct language samples (monolingual and bilingual). The Ngöbe adult sample exhibited more clustering variability, which may speak to the presence of multiple cultural models of agency.

Individual differences in consensus model membership. Ngöbe adults. The clustering results indicate substantial within-culture variability among Ngöbe. Based on prior research, we anticipated that Ngöbe members of the ecocentric Model 2 would also lead more traditional community-based lifestyles, as indexed by five measures (livelihood, Indigenous church membership, age, language identity, formal schooling). Of these variables, livelihood and language identity reliably distinguished between Ngöbe clustered with Model 1 and Model 2. Members of Model 2 were comparatively more likely to be engaged in subsistence activities versus external occupations (e.g., wage-earning work), $F(1,19)=4.28, p=.05, \eta^{2}=$ .18 , and to identify Ngöbere as their primary language rather than Spanish, $F(1,19)=9.90, p<.01, \eta^{2}=$ $.34 .^{26}$

Model 2 members were also more likely to be in the relational condition, $F(1,19)=7.80, p<.05, \eta^{2}$ $=.29$. This could be interpreted as further evidence that the relational framing of agency facilitated an ecocentric framework of agency that is widely available in the Ngöbe community. At the same time, the characteristics of Model 2 may also be partially due to the experimental condition. Sample sizes do not permit us to factor out the influence of condition on consensus models.

US college students. Among US participants, there were no reliable differences between participants clustered with Model 1 versus Model 2 for experimental condition, task language, or

\footnotetext{
${ }^{24}$ Recall that ranks are reverse scored, hence the negative correlation between expertise and higher ranks.

${ }^{25}$ In practice this often meant that Model 1 experts increased the distance between humans and animals in their experience rankings, relative to less expert participants. Interestingly, the specific items of highest difficulty (and lower rank) from the animal category were the bird and frog, consistent with an anthropocentric model that places mammals closer to the human prototype than non-mammals.

${ }^{26}$ Due to missing demographic information, Ngöbe $n=21$ for these analyses.
} 
individual characteristics. This analysis, however, is limited by the small number of US participants clustered with Model $2(n=4)$.

Conceptual differences in cross-model membership. Given that a cultural majority emerged for each conceptual model, we were interested in the responses of those minority individuals who were crossclustered with the other consensus model (i.e., that of their non-majority culture). The agency attribution profiles of these subgroups could speak to what happens at the boundaries of each conceptual model. All differences reported below were significant at the $p<.05$ level in MANOVAs (either by culture or model membership) comparing agency attributions for cognition and experience.

The four US participants who clustered with the Ngöbe-majority Model 2 differed from their US Model 1 counterparts in their treatment of plants (more experience and cognition) and complex artifacts (less cognition). They remained distinct from Ngöbe members of Model 2, however, in their rejection of cognition for abiotic kinds. The eight Ngöbe participants who clustered with the US-majority Model 1 diverged from their Ngöbe Model 2 counterparts in their attributions to plants and abiotic kinds (less agency to both, which held at or near significance for both experience and cognition). However, they remained distinct from US members of Model 1 by attributing more cognition to abiotic kinds and less cognition to complex artifacts.

These findings suggest that the generally anthropocentric view of agency among US college students may afford variation toward a more biology-based model that encompasses plants but rejects anything considered inanimate. Conversely, the generally ecocentric view of agency among Ngöbe individuals may afford variation toward a more animal-centric model where the agency of non-animal natural kinds is relatively diminished.

Individual differences in within-group competence. We next analyzed individual differences associated with competence scores, which indicate how well an individual fits the consensus model to which they were assigned. Analyses were run separately for Model 1 and Model 2 scores within each cultural group (Ngöbe or US) given that demographic features were hardly comparable between groups.

Ngöbe adults. We anticipated that competence scores for Ngöbe participants clustered with Model $2(n=14)$ would correlate with more traditional Ngöbe lifestyles, given that this model reflects an ecocentric orientation to agency. Indeed, greater competence was reliably correlated with greater age $(r=$ $.64, p<.05)$, less formal schooling $(r=-.73, p<.01)$, and, unexpectedly, gender (males more expert, $r=.57$, $p<.05$ ). This supports the idea that the ecocentric model of agency is facilitated by greater engagement with Ngöbe cultural knowledge (e.g., being an elder) and/or less involvement with non-Ngöbe cultural discourses (e.g., Western style schooling). There were no reliable correlations between competence and individual characteristics among Ngöbe clustered with Model $1(n=8)$, but the modest sample size limited this analysis.

US college students. We had no hypotheses concerning individual predictors of competence scores among US participants beyond the possibility (based on findings reported in ojalehto, et al., 2017) that religious belief or experience with nature may be relevant to notions about plants. Among US participants clustered with the anthropocentrically oriented Model $1(n=31)$, grade level was positively correlated with greater competence $(r=.37, p<.05)$. This may suggest that university schooling (possibly psychology-specific, given that these participants were drawn from a psychology subject pool) strengthens the dominant cultural model of agency. Unexpectedly, competence for US members of Model $2(n=4)$ was negatively related to religion (in contrast to our prior findings), such that those reporting fewer religious beliefs were more competent $(r=-.96, p<.05) .{ }^{27}$ The small sample prohibits firm conclusions, and both these relationships should be interpreted with considerable caution given the lack of predictions.

${ }^{27}$ Religiosity was a composite measure of the three religious belief items, see Appendix B1. 


\subsection{Experiment 2: Discussion}

Two distinct cultural conceptual frameworks for agency are evident in these results. The model most salient among Ngöbe informants offers an ecocentric perspective on which plants and abiotic kinds are both significantly more agentic than complex artifacts, and plants in particular figure as robust agents. In contrast, the US college student model offers an anthropocentric perspective that includes only humans and animals as agents proper, while complex artifacts and plants are attributed limited degrees of cognitive or experiential agency respectively. US college results held across Spanish and English task versions so these cross-cultural results do not simply reflect cross-linguistic differences.

To test our proposal that these agency attribution models reflect distinct grounds for inferring agency, we introduced an experimental manipulation and probed the conceptual dimensions underlying agency attribution. The experimental relational framing of agency facilitated greater agency attributions for Ngöbe participants only, indicating that relational principles are important to inferring agency on this cultural model. Further evidence for diverse inferential principles came from analysis of conceptual dimensions underlying cultural models of agency. US college results were consistent with a twodimensional mind perception framework where agency is conceptualized separately according to experiential or cognitive capacities, and cognition in particular is decoupled from animacy judgments. Ngöbe results were consistent with a more unified conceptual framework for agency where the experience-cognition distinction is less pronounced and both dimensions track together with a third dimension of animacy. These results suggest that both groups work from a biology-based model of experience, but the dimension labeled cognition taps into distinct notions of agency as a representational capacity separable from animacy (seen in the vast majority of US students), or as an ecological capacity linked to both animate force and experiential capacity (most common among the Ngöbe).

Cultural consensus modeling allowed us to identify two latent conceptual models underlying these cultural differences and to analyze the conceptual properties and individual characteristics associated with each model. Three key findings emerge. First, beyond the overall agreement on the agency of animal kinds, two separate consensus models were detected, confirming that ecocentric and anthropocentric attribution patterns represent distinct conceptual models for understanding non-animal agency. Second, forms of competence associated with each model provided converging evidence for the hypothesized cultural frameworks in terms of conceptual content (items that predicted competence on the anthropocentric and ecocentric models were computerized artifacts and abiotic natural kinds, respectively) and individual variability (competence on each model was associated with university schooling or traditional cultural engagement, respectively). Third, these modeling results suggest that there are multiple models of agency available in the Ngöbe community, with the more pronounced cultural model being an ecocentric model of agency. The US college student sample reached strong convergence on a single dominant model of anthropocentric agency.

The CCM findings underscore how conceptual behavior is embedded in systems of knowledge and practice. Among Ngöbe informants, for instance, the ecocentric model of agency correlates with greater engagement in Ngöbe cultural practices (e.g., language, livelihood) and less engagement in Westernized cultural discourses (e.g., formal schooling). While this may be read as suggesting that Westernization leads to cultural homogenization, we would caution against a simple "replacement" model. CCM presents a (statistically necessary) two-model simplification but blended models might also appear, as observed for Ngöbe participants who clustered with the anthropocentric Model 1 but remained more ecocentric in their attributions to abiotic kinds and complex artifacts than US Model 1 members (likewise, a blended model was observed for US cross-model members). Such nuanced agency attribution profiles attest to the complexity of agency frameworks. More broadly, our CCM analysis offers a methodological contribution to cultural cognition studies by introducing a bottom-up modeling approach that goes beyond group-level differences to identify multiple conceptual models and their individual correlates within and across groups. 


\section{General discussion}

Across two experiments, multiple converging measures paint a robust picture of distinct cultural frameworks for understanding agency. Ngöbe adults were consistently more likely to attribute agency to plants and abiotic kinds than US college students, who in turn were more likely to attribute agency to complex computerized artifacts. We argue that these disparate models point to culturally distinct grounds for inferring agency as a relational capacity understood on an ecocentric prototype (folkcommunication), or as a psychological capacity understood on an anthropocentric prototype (folkpsychology). The current experiments provided several forms of evidence to support this proposal. First, analysis of explanations showed that US participants interpret agency as a scalar, prototypically human capacity requiring consciousness. Ngöbe interpreted agency in terms of directed interactions with the environment and others, indicating a concern for the relational dimensions of agency. Second, by introducing a relational framing of capacity probes we demonstrated that Ngöbe (but not US) agency attributions are facilitated by a relational perspective, consistent with our claim that the capacity to relate is a grounding principle for inferring agency on folkcommunication.

Third, the conceptual organization of agency dimensions differed across cultures. US college student attributions suggested a domain-specific approach whereby animacy followed a biology-based model, experience followed a narrower biological model that mostly excluded plants, and cognition followed an artifact kinds model that excluded plants but included computerized artifacts. That US models diverged across these dimensions indicates that distinct inferential principles are at stake in each domain. The cognition dimension especially points to an anthropocentric framework where agency is understood as a representational capacity on a human prototype. By contrast, the conceptual organization observed for Ngöbe agency attributions presents a more unified framework for agency. All three dimensions fit an overarching natural kinds model within which experience was more biologybased while cognition and animacy were more ecology-based. This suggests that for Ngöbe adults the grounding principles for inferring agency are similar across all three domains, consistent with our proposal that folkcommunication provides a broad framework for conceptualizing agency as a capacity to relate with others and the environment.

Finally, we consolidated these findings using a new method for analyzing cognitive variability through a hierarchical Bayesian inferential approach to cultural consensus modeling. With culture treated as a latent variable, bottom-up modeling results confirmed that the observed cultural differences in agency attribution arise from two distinct bodies of consensus knowledge rather than deviations from a single model. The two consensus models were consistent with the hypothesized anthropocentric versus ecocentric prototypes for agency. Individual membership on each model largely clustered with actual cultural membership, accompanied by predictable patterns of cross- and within-culture variability in conceptual content and forms of competence. Ngöbe participants appeared to have access to multiple models of agency as ecocentric (the more pronounced cultural model) or relatively animal-centric, while US college participants reached strong consensus on a single model of agency that reflects an anthropocentric orientation.

\section{Limitations}

Before discussing the implications of these findings we address potential limitations. One potential concern arises with our experimental condition. Perhaps by including an object following a capacity predicate in the relational condition, we implied that the capacity in question properly applies to the subject (i.e., asking if plants can "feel hunger for nutrients" may imply that plants can feel hunger), and perhaps Ngöbe participants are more sensitive to this implicature than US participants. However, we would argue that this pragmatic implicature (such as it is) is conceptually aligned with our argument for a relational agency framework. If one's focus is on the interaction between subject and object, then agency predicates do in fact play the conceptual role of binding two entities in a particular kind of relationship 
(see also Knobe \& Prinz, 2008). To the extent that the predicate-object pairing accentuates the relational aspect of agency, this can be interpreted as a meaningful property of a folkcommunication framework. In any case, the key cultural differences also hold for the original condition so any interpretive questions surrounding the relational condition would not explain away our results.

Cultural consensus modeling indicated the presence of multiple models in our Ngöbe sample, contrasting with strong agreement on a single model in our US college sample. One might worry that this finding may reflect a (presumed) greater range of individual variability among the Ngöbe adult sample (age, schooling, etc.) as compared to our US college student sample which was simply too homogenous to yield multiple models. However, it is important to note that our US participants did vary on potentially important characteristics such as bilingualism, religiosity, and experience with nature, but they nonetheless presented a single dominant cultural model. This raises important questions for further research concerning the kinds of variability that can support the presence of multiple models within a community. For instance, it would be informative to explore how ecological experience may (or may not) influence agency concepts among diverse Western samples beyond the college classroom and beyond the US, as we are currently doing in follow-up research.

Sample generalizability. Having shown that the US college student model of agency does not extend to Ngöbe adults, one may question whether US college students are even representative of the broader US population. Yet this is the sample on which the vast majority of research in this area has been conducted (for a few examples of many such studies, see Arico et al., 2011; Gutheil, et al., 1998; Opfer, 2002; Opfer \& Gelman, 2001). Given this history it is a reasonable first step to use college students as a baseline but then to build on this in further research exploring US within-culture variability.

At the same time using "Ngöbe adults" to describe our Panamanian sample may imply generalizability that remains to be demonstrated. Within Panama, Ngöbe experience diverse environments, livelihoods and westernization forces. Even within our current sample we found evidence for two distinct cultural models. This resonates with the anthropological literature indicating that Indigenous models of agency are widespread but also widely diverse, implying that Ngöbe culture supports an ecocentric model of agency rather than requiring it for cultural membership.

Cross-cultural difference generalizability. It is also important to point out that our two national samples differed in many ways including formal schooling, economic status, and (presumably) personal familiarity with complex artifacts, plants, and non-urban ecosystems. From one perspective, this might raise concerns that certain variables-e.g., familiarity with complex artifacts, formal schooling experience-may (partly) explain the observed cultural differences in agency attribution. From another perspective, however, each factor reflects and reinforces cultural epistemologies. Following this approach we would argue that a research agenda that aims to "partial out" all such differences in this manner risks losing sight of what is at stake (see Rogoff \& Angelillo, 2002 for an extended treatment of this issue).

For example, one potential concern is that differential familiarity with complex artifacts (robots and computers) may explain the observed differences between Ngöbe and US attributions on these items. These cross-cultural differences were most evident on the cognition dimension and we interpreted this to indicate that Ngöbe and US participants infer agency on this dimension based on distinct principles. ${ }^{28}$ But conceivably this could also reflect variable knowledge about such artifacts. As noted earlier, many complex artifacts including laptop computers are now common in the Ngöbe community, but robots are present only in media representations. To the extent that Ngöbe informants are unfamiliar with the functional interactive capacities of robots, this could lead them to be less likely than US college students

${ }^{28}$ The results for complex artifacts remain somewhat inconclusive. In Experiment 2, we observed a reliable trend for US participants to attribute greater agency to complex artifacts as compared to Ngöbe. However, Experiment 1 findings for complex artifacts were mixed (US rankings were higher as expected, but inclusions were similar across both groups). Further work is needed to clarify the extent of the difference. 
1064

1065

1066

1067

1068

1069

1070

1071

1072

1073

1074

1075

1076

1077

1078

1079

1080

1081

1082

1083

1084

1085

1086

1087

1088

1089

1090

1091

1092

1093

1094

1095

1096

1097

1098

1099

1100

1101

1102

1103

1104

1105

1106

1107

1108 to ascribe agency to robots (for more on functional ascriptions of agency, see Knobe \& Prinz, 2008). ${ }^{29}$ This may be the case and it would be consonant with an ecocentric approach to agency based on relational principles. (However, it is unlikely to explain our findings for agency ascription to computers given that they are fairly common in the Ngöbe community.) Even though complex artifacts are now commonplace, certain Ngöbe individuals such as elders or subsistence farmers may use such artifacts less frequently than others. This points to the larger issue that familiarity with complex artifacts is itself a cultural factor that may relate to any number of lifestyle choices. The same logic holds for formal schooling, which was inversely correlated with an ecocentric model of agency among Ngöbe informants.

Given the complexity of cultural systems, it may not be feasible (or desirable) to isolate one factor and give it explanatory priority in a system where many variables interrelate. For this reason, consensus modeling is a felicitous tool for studying cognitive diversity because it reduces reliance on a priori cultural categories in favor of exploring multiple forms of variability within and across conceptual models. Using this tool, we found that cultural lifestyle factors do converge predictably with forms of competence on both ecocentric and anthropocentric models of agency. Such findings suggest that cultural factors such as schooling or familiarity with complex artifacts contribute to diverse systems of organizing conceptual knowledge, rather than serving as nagging confounds that detract from understanding of "pure" cultural differences.

\section{Interpretation of cultural differences}

Our findings bear on theoretical debates surrounding animacy, cultural conceptual domains, and the role of folkpsychology in cognition more broadly. With respect to animacy, the dominant approach in cultural psychology has been to assume that Indigenous attributions of agency to nonhumans represent animistic overextensions of folkpsychology (or perhaps metaphorical extensions of agency). Taken alone, our agency attribution findings could be consistent with such an overextension account on which the two cultural groups mobilize a similar (universal) concept of agency but extend it at different rates to different non-animal kinds. On this account, one might argue that Ngöbe and US frameworks share a basic concept of agency with one cultural framework more restrictive than the other, or one framework overextended relative to the other.

However, the full picture of results challenges a simple overextension account by pointing to more complex forms of diversity. For one, US and Ngöbe agency attributions to complex artifacts versus natural non-animal kinds go in opposite directions. This undermines the claim that Ngöbe agency concepts can be explained as (metaphorical) overextensions of folkpsychology, which would predict a main effect for kind while we observed an interaction of culture with abiotic kinds versus complex artifacts. Additionally, our results point to distinctive cultural organization of agency concepts across the conceptual dimensions of experience, cognition, and animacy. This indicates that the conceptual domain of agency is structured according to different inferential principles in each culture, rather than being based on universal folkpsychological principles that extend to different targets across cultures. Our bottom-up consensus modeling results confirmed that two distinct and coherent patterns of reasoning underlie these cross-cultural differences, rather than a single model accompanied by deviations as would be expected on the overextension account. Finally, Ngöbe and US participants' explanations attested to different forms of reasoning about agency and the importance of relational reasoning to the Ngöbe framework was confirmed experimentally through a framing manipulation.

Considered as a whole, the evidence lends itself most readily to explanation in terms of distinct principles for inferring agency, leading to cultural variability in the conceptual structure of the agency domain. For US college students, the overall picture suggests a domain-specific profile that holds animate kinds and psychological kinds in different (but overlapping) categories, and infers distinct dimensions of

${ }^{29}$ We thank an anonymous reviewer for pointing out this possibility. 
folkpsychological agency on the basis of either a biological capacity for experience or a representational capacity for cognition. For Ngöbe adult informants, the overall picture suggests an integrated conceptual framework that holds diverse natural kinds under a common domain of folkcommunicative agency, and infers a variety of agency capacities (experience, cognition, and animacy) on the basis of similar principles-principles that we propose are fundamentally relational in nature. Supporting this proposal, in other recent experiments we find that cultural frameworks for agency carry cognitive consequences for inferential reasoning about the actual behavior of nonhuman agents (ojalehto, et al., 2017; ojalehto, et al., 2015). For instance, Ngöbe perspectives afford insights into nonhuman behaviors such as plant communication and kin altruism that have only recently been discovered in Western life sciences. Considering these empirical results together with the broader literature on Indigenous relational epistemologies, we argue that Ngöbe conceptual frameworks challenge the widespread assumption that anthropocentric folkpsychology is a universal organizing principle for the conceptual domain of agency. Interestingly, a sizable minority of US respondents were willing to ascribe experiential capacities to plants. This dovetails with our findings from recent work showing that US college students are willing to ascribe certain basic experiential capacities such as feeling (but not feeling pain) to plants (ojalehto, et al., 2017). This suggests an appreciation of sentience not strictly tied to having a brain, although prior research based on domain-specificity theory has rarely considered this possibility (or considered it a surprise when results indicated such) (e.g., Arico, et al., 2011; Opfer \& Gelman, 2001). This intriguing observation remains to be explored more fully

These findings raise a wealth of questions about the interaction of agency concepts and broader cultural systems (ojalehto \& Medin, 2015). For instance, one might speculate that the US folkpsychological stance on agency is tied to a heightened focus on (human) minds under Cartesian dualism, whereas the Ngöbe ecological stance on agency partakes of a cultural worldview that sees humans as part of nature (Kimmerer, 2013; Medin \& Bang, 2014). In fact, Western researchers working from an anthropocentric folkpsychology may have mischaracterized Indigenous concepts by claiming that they represent a supernatural or anthropomorphic overextension of human-like mental-state concepts to non-animal kinds. If one is not working from a human psychological prototype, then the view that nonhuman behavior reflects intentional agency is quite plausible. Indeed, this is precisely the message of a growing body of scientific research on the intelligent behavior of plants, bacteria, and other natural kinds, including even some abiotic forces (Calvo \& Keijzer, 2011; Chamovitz, 2012; Haila \& Dyke, 2006; Kauffman, 2008; Marder, 2013; Narby, 2006; Trewavas, 2016). Our proposal that such forms of agency may be interpreted under a folkcommunication framework accords with a wide body of psychological research that shows how people-including typical Western samples-organize concepts of agency around relational and social dimensions (e.g., Hirschfeld, 2013; Luhrmann, 2011; Mascaro \& Csibra, 2012; ojalehto, et al., 2013; Tamir, et al., 2016). Underscoring the role of relational categories in cognition more generally (Gentner \& Kurtz, 2005), especially among non-Western cultures (Nisbett, et al., 2001), folkcommunication offers an important new theoretical perspective on agency and domain-specific knowledge organization that is based on the relational epistemologies of Indigenous communities.

\section{Conclusion}

We have argued that Ngöbe individuals have access to an ecocentric conceptual framework for agency that is fundamentally geared toward understanding interactions and relationships, one that differs from the US anthropocentric folkpsychology focused on internalized mental states. Our findings suggest that grounding principles for inferring agency diverge across cultures, leading to patterns of agency attribution that cut across different domains and track different conceptual dimensions rather than fitting a neat animistic overextension account of folkpsychology to non-animal kinds. While an

1154 emphasis on psychological agency as distinct from other (biological, physical) forms of agency has been 
1155 widely assumed to be a conceptual prior, these findings suggest it may instead be a feature of Western 1156 cultural epistemologies.

1157 The implications of our account are potentially far-reaching, given that agency concepts play a 1158 fundamental role in cognitive processes ranging from causality and mind perception to morality (e.g., 1159 Banaji \& Gelman, 2013). The present research offers a novel perspective on the universality of domain1160 specific folktheories for agency and resonates with calls to reevaluate the privileged role of mentalistic 1161 folkpsychology in social cognition (Heyes, 2014; Hirschfeld, 2013). Furthermore, human-nature 1162 relationships are currently under tremendous stress as a result of Western worldviews and ways of 1163 interacting with the rest of nature (Kahn et al., 2010; Mace, 2014). Ngöbe perspectives could be useful in 1164 offering a way to understand agency based on an ecologically inclusive principle of relational capacity, 1165 providing a complementary perspective to the common Western narrative of human-centered agency. 1166 Given that our intuitive recognition of other kinds as agents helps to shape their role in our social and 1167 moral communities, it seems well worth exploring a conceptual framework for ecocentric agency. 


\section{Acknowledgements}

1170 For comments and conversations about ideas presented in this article, we are thankful to Eugene

1171 Anderson, Scott Atran, Rumen Iliev, Joshua Knobe, Olivier Le Guen, Jonas Nagel, Jeremy Narby, Ara

1172 Norenzayan, Jeremy Ojalehto, Lance Rips, Sonya Sachdeva, Fernando Santos-Granero, Rebecca Seligman,

1173 Sandra Waxman, the late Philip Young, and fellow Mosaic Lab members. Thanks also to three

1174 anonymous reviewers for their constructive feedback. For statistical and programming contributions, we

1175 thank Royce Anders, Pascal Paschos, and Satoru Suzuki. For their contributions to data collection and

1176 analysis we thank Linda Flores, Yereida Gallardo, Melissa Vega, Osbeyda Navarette, Elaine Aedo, and

1177 Lan Nguyen. Versions of this article were presented at ZiF research group on the Cultural Constitution of

1178 Causal Cognition, CogSci Meetings, and the Society for Anthropological Science. This work was

1179 supported by a DRMS Doctoral Dissertation Research Improvement Grant 1427035 and National Science

1180 Foundation grants SES0962185 and DRL1114530 and AFOSR FA9550-14-1-0030, and several grants from

1181 Northwestern University. 
1182

1183

1184

1185

1186

1187

1188

1189

1190

1191

1192

1193

1194

1195

1196

1197

1198

1199

1200

1201

1202

1203

1204

1205

1206

1207

1208

1209

1210

1211

1212

1213

1214

1215

1216

1217

1218

1219

1220

1221

1222

1223

\section{References}

Anders, R. (2013). CCTpack: Cultural Consensus Theory applications to data. R package version 1.2.

Anders, R. (2014). Package "CCTpack" Manual. Retrieved from http://citeseerx.ist.psu.edu/viewdoc/download?doi=10.1.1.649.4064\&rep=rep1\&type=pdf

Anders, R., \& Batchelder, W. H. (2012). Cultural consensus theory for multiple consensus truths. Journal of Mathematical Psychology, 56(6), 452-469.

Anders, R., Oravecz, Z., \& Batchelder, W. H. (2014). Cultural consensus theory for continuous responses: a latent appraisal model for information pooling. Journal of Mathematical Psychology, 61, 1-13.

Arico, A., Fiala, B., Goldberg, R. F., \& Nichols, S. (2011). The Folk Psychology of Consciousness*. Mind E Language, 26(3), 327-352.

Astuti, R. (2001). Are We all Natural Dualists? A Cognitive Developmental Approach*. Journal of the Royal Anthropological Institute, 7(3), 429-447.

Astuti, R., Solomon, G. E., \& Carey, S. (2004). Constraints on conceptual development: A case study of the acquisition of folkbiological and folksociological knowledge in Madagascar. Monographs of the Society for Research in Child Development, 69(3), i-161.

Atran, S., \& Medin, D. L. (2008). The native mind and the cultural construction of nature: MIT Press Cambridge.

Atran, S., \& Norenzayan, A. (2004). Religion's evolutionary landscape: Counterintuition, commitment, compassion, communion. Behavioral and brain sciences, 27(06), 713-730.

Banaji, M., \& Gelman, S. A. (2013). Navigating the Social World: What infants, children, and other species can teach us. Oxford, UK: Oxford University Press.

Barrett, H. C., Todd, P. M., Miller, G. F., \& Blythe, P. W. (2005). Accurate judgments of intention from motion cues alone: A cross-cultural study. Evolution and Human Behavior, 26(4), 313331.

Barrett, J. L. (2000). Exploring the natural foundations of religion. Trends in Cognitive Sciences, 4(1), 29-34.

Bird-David, N. (1999). “Animism” Revisited: Personhood, Environment, and Relational Epistemology 1. Current Anthropology, 40(S1), S67-S91.

Boyer, P. (1996). What makes anthropomorphism natural: Intuitive ontology and cultural representations. Journal of the Royal Anthropological Institute, 83-97.

Boyer, P. (2003). Religious thought and behaviour as by-products of brain function. Trends in Cognitive Sciences, 7(3), 119-124.

Bray, D. B., Ellis, E. A., Armijo-Canto, N., \& Beck, C. T. (2004). The institutional drivers of sustainable landscapes: a case study of the 'Mayan Zone'in Quintana Roo, Mexico. Land Use Policy, 21(4), 333-346.

Calvo, P., \& Keijzer, F. (2011). Plants: adaptive behavior, root-brains, and minimal cognition. Adaptive Behavior, 19(3), 155-171.

Carey, S. (2009). The origin of concepts. Oxford, UK: Oxford University Press.

Chamovitz, D. (2012). What a plant knows: a field guide to the senses: Macmillan. 
1224

1225

1226

1227

1228

1229

1230

1231

1232

1233

1234

1235

1236

1237

1238

1239

1240

1241

1242

1243

1244

1245

1246

1247

1248

1249

1250

1251

1252

1253

1254

1255

1256

1257

1258

1259

1260

1261

1262

1263

1264

1265

1266

Csibra, G., \& Gergely, G. (2007). 'Obsessed with goals': Functions and mechanisms of teleological interpretation of actions in humans. Acta psychologica, 124(1), 60-78.

Danziger, E., \& Rumsey, A. (2013). Introduction: From Opacity to intersubjectivity across languages and cultures. Language and Communication, 33(3), 247-250.

Gelman, S. A., \& Legare, C. H. (2011). Concepts and folk theories. Annual review of anthropology, 40, 379-398.

Gentner, D., \& Kurtz, K. J. (2005). Relational categories. Categorization inside and outside the lab, 151-175.

Gjording, C. N. (1991). Conditions not of their choosing: The Guaymi Indians and mining in multinationals in Panama Washington, D.C.: Smithsonian Institution Press.

Gordon, B. L. (1982). A Panama forest and shore: natural history and Amerindian culture in Bocas del Toro: Pacific Grove: California, Boxwood Press.

Gray, H., Gray, K., \& Wegner, D. M. (2007). Dimensions of mind perception. Science, 315(5812), 619-619.

Gutheil, G., Vera, A., \& Keil, F. C. (1998). Do houseflies think? Patterns of induction and biological beliefs in development. Cognition, 66(1), 33-49.

Guthrie, S., Agassi, J., Andriolo, K. R., Buchdahl, D., Earhart, H. B., Greenberg, M., ... Sharpe, K. J. (1980). A Cognitive Theory of Religion [and Comments and Reply]. Current Anthropology, 181-203.

Haila, Y., \& Dyke, C. (2006). How nature speaks: the dynamics of the human ecological condition. Durham, NC: Duke University Press.

Hall, M. (2011). Suny Series on Religion and the Environment: Plants As Persons: a Philosophical Botany: SUNY Press.

Hallowell, A. I. (1960). Ojibwa ontology, behavior, and world view Readings in indigenous religions (pp. 18-49).

Harvey, G. (2005). Animism: Respecting the living world. Kent Town, South Australia: Wakefield Press.

Heyes, C. (2014). Submentalizing I Am Not Really Reading Your Mind. Perspectives on Psychological Science, 9(2), 131-143.

Hirschfeld, L. A. (2013). The myth of mentalizing and the primacy of folk sociology. In M. Banaji \& S. A. Gelman (Eds.), Navigating the Social World: What Infants, Children, and Other Species Can Teach Us (pp. 101-106). Oxford, UK: Oxford University Press.

Inagaki, K., \& Sugiyama, K. (1988). Attributing human characteristics: Developmental changes in over-and underattribution. Cognitive Development, 3(1), 55-70.

Ingold, T. (2006). Rethinking the animate, re-animating thought. Ethnos, 71(1), 9-20.

Ingold, T. (2011). Being alive: Essays on movement, knowledge and description: Taylor \& Francis.

Johnson, S. C. (2003). Detecting agents. Philosophical Transactions of the Royal Society of London. Series B: Biological Sciences, 358(1431), 549-559.

Kahn, P. H., Ruckert, J. H., Severson, R. L., Reichert, A. L., \& Fowler, E. (2010). A nature language: An agenda to catalog, save, and recover patterns of human-nature interaction. Ecopsychology, 2(2), 59-66.

Kauffman, S. A. (2008). Reinventing the sacred: A new view of science, reason, and religion: Basic Books. 
Kimmerer, R. W. (2013). Braiding sweetgrass: indigenous wisdom, scientific knowledge and the teachings of plants. Minneapolis, MN: Milkweed Editions.

Knobe, J. (2011). Finding the mind in the body. In M. Brockman (Ed.), Future Science: Essays from the Cuttting Edge (pp. 184-196). New York: Random House.

Knobe, J., \& Prinz, J. (2008). Intuitions about consciousness: Experimental studies. Phenomenology and the cognitive sciences, 7(1), 67-83.

Kohn, E. (2007). How dogs dream: Amazonian natures and the politics of transspecies engagement. American ethnologist, 34(1), 3-24.

Kohn, E. (2013). How Forests Think: Toward an Anthropology beyond the Human: Univ of California Press.

Le Guen, O., Iliev, R., Lois, X., Atran, S., \& Medin, D. L. (2013). A garden experiment revisited: inter-generational change in environmental perception and management of the Maya Lowlands, Guatemala. Journal of the Royal Anthropological Institute, 19(4), 771-794.

Lee, M. D., Steyvers, M., De Young, M., \& Miller, B. (2012). Inferring expertise in knowledge and prediction ranking tasks. Topics in cognitive science, 4(1), 151-163.

Lee, M. D., Steyvers, M., \& Miller, B. (2014). A cognitive model for aggregating people's rankings. PloS one, 9(5), e96431.

Lewis, M. (1990). The development of intentionality and the role of consciousness. Psychological Inquiry, 1(3), 230-247.

Lowder, M. W., \& Gordon, P. C. (2015). Natural forces as agents: Reconceptualizing the animate-inanimate distinction. Cognition, 136, 85-90.

Luhrmann, T. (2011). Toward an anthropological theory of mind (Overview). Suomen Antropol. J. Finn. Anthropol. Soc, 36, 5-69.

Mace, G. M. (2014). Whose conservation? Science, 345(6204), 1558-1560.

Marder, M. (2013). Plant intelligence and attention. Plant Signaling \& Behavior, 8(5), e23902. doi: 10.4161/psb.23902

Mascaro, O., \& Csibra, G. (2012). Representation of stable social dominance relations by human infants. Proceedings of the National Academy of Sciences, 109(18), 6862-6867.

Mayhew, C., Jordan, O., Panamá, D., Argentina, V., \& Rolnick, A. (2010). Panama is in Breach of its Obligations to Indigenous Peoples under the Convention on the Elimination of All Forms of Racial Discrimination Report to: United Nations Committee on the Elimination of Racial Discrimination, on the occasion of its consideration of the 15th to 20th Periodic Reports of Panama. Panama: Asociación para la Conservacion y el Desarrollo (ACD).

Medin, D. L., \& Bang, M. (2014). The cultural side of science communication. Proceedings of the National Academy of Sciences, 111(Supplement 4), 13621-13626.

Medin, D. L., ojalehto, b., Marin, A., \& Bang, M. (2013). Culture and epistemologies: putting culture back into the ecosystem. In M. Gelfand, C. Chiu \& Y.-Y. Hong (Eds.), Advances in culture and psychology (Vol. 4, pp. 177-217). Oxford, UK: Oxford University Press.

Medin, D. L., Ross, N., Cox, D., \& Atran, S. (2007). Why folkbiology matters: Resource conflict despite shared goals and knowledge. Human Ecology, 35(3), 315-329.

Montezuma, L. P. (1991). Nun Roae Kugwe Kira Niebare Nuen: Nuestros Abuelos Nos Contaron Historias (Our Grandparents Told Us These Stories). Comarca Ngobe, Panama: Accion Cultural Ngobe. 
1310

1311

1312

1313

1314

1315

1316

1317

1318

1319

1320

1321

1322

1323

1324

1325

1326

1327

1328

1329

1330

1331

1332

1333

1334

1335

1336

1337

1338

1339

1340

1341

1342

1343

1344

1345

1346

1347

1348

1349

1350

1351

Narby, J. (2006). Intelligence in nature: An inquiry into knowledge: Penguin. com.

Nisbett, R. E., Peng, K., Choi, I., \& Norenzayan, A. (2001). Culture and systems of thought: holistic versus analytic cognition. Psychological review, 108(2), 291-310.

ojalehto, b., Medin, D., \& Garcia, S. (2017). Conceptualizing agency: Folkpsychological and folkcommunicative perspectives on plants. Cognition, 162, 103-123.

ojalehto, b., Medin, D., Horton, W., Garcia, S., \& Kays, E. (2015). Seeing cooperation or competition: Ecological interactions in cultural perspectives. Topics in Cognitive Science(Anthropological perspectives on cognition), 1-22. doi: 10.1111/tops.12156

ojalehto, b., Waxman, S. R., \& Medin, D. L. (2013). Teleological reasoning about nature: intentional design or relational perspectives? Trends in Cognitive Sciences, 17(4), 166-171.

Opfer, J. E. (2002). Identifying living and sentient kinds from dynamic information: The case of goal-directed versus aimless autonomous movement in conceptual change. Cognition, 86(2), 97-122.

Opfer, J. E., \& Gelman, S. A. (2001). Children's and Adults' Models for Predicting Teleological Action: The Development of a Biology-Based Model. Child development, 72(5), 1367-1381.

Opfer, J. E., \& Siegler, R. S. (2004). Revisiting preschoolers' "living things" concept: A microgenetic analysis of conceptual change in basic biology. Cognitive psychology, 49(4), 301-332.

Pierotti, R. (2011). Indigenous knowledge, ecology, and evolutionary biology. New York: Routledge: Taylor \& Francis Group.

Reid, A. (1994). Diccionario Guaymi-Espanol-Ingles Diccionario Guaymi-Espanol-Ingles. Panama: Asamblea Espiritual Nacional de los Baha'is de Panama.

Rogoff, B., \& Angelillo, C. (2002). Investigating the coordinated functioning of multifaceted cultural practices in human development. Human Development, 45(4), 211-225.

Romney, A. K., Weller, S. C., \& Batchelder, W. H. (1986). Culture as consensus: A theory of culture and informant accuracy. American anthropologist, 88(2), 313-338.

Sarsaneda del Cid, J. (2009). Ni Ngóbe Tó Blitde Ño: Cómo Hablan Los Ngóbe. Panama: Acción Cultural Ngóbe.

Spelke, E. S., \& Kinzler, K. D. (2007). Core knowledge. Developmental science, 10(1), 89-96.

Sperber, D., \& Hirschfeld, L. A. (2004). The cognitive foundations of cultural stability and diversity. Trends in cognitive sciences, 8(1), 40-46.

Strickland, B., Silver, I., \& Keil, F. C. (2016). The texture of causal construals: Domain-specific biases shape causal inferences from discourse. Memory $\mathcal{E}$ Cognition, 1-14.

Tamir, D. I., Thornton, M. A., Contreras, J. M., \& Mitchell, J. P. (2016). Neural evidence that three dimensions organize mental state representation: Rationality, social impact, and valence. Proceedings of the National Academy of Sciences, 113(1), 194-199.

Trewavas, A. (2016). Intelligence, Cognition, and Language of Green Plants. Frontiers in psychology, 7.

Walker, H. (2013). Under a Watchful Eye: Self, Power, and Intimacy in Amazonia. Berkeley, CA: University of California Press.

Waytz, A., Gray, K., Epley, N., \& Wegner, D. M. (2010). Causes and consequences of mind perception. Trends in cognitive sciences, 14(8), 383-388. 
1352 Wellman, H. M., \& Gelman, S. A. (1992). Cognitive development: Foundational theories of core

1353

1354

1355

1356

1357

1358

1359

1360 domains. Annual review of psychology, 43(1), 337-375.

Young, P. D. (1975). Guaymi nativism: its rise and demise. Paper presented at the XLI International Congress of Americanists.

Young, P. D., \& Bort, J. R. (1999). Ngobe adaptive responses to globalization in Panama. In W. M. Loker (Ed.), Globalization and the Rural Poor in Latin America (pp. 111-136). Boulder, CO: Lynne Rienner. 


\section{APPENDICES}

\section{Appendix A: Experiment 1}

\section{A1. Methods and procedure}

The present task was adapted from the original mind perception survey in several ways: (1) Response method was ranking rather than pairwise comparisons; (2) Items included plants and abiotic kinds in addition to selected original items; (3) Only a subset of capacity probes was used; and (4) Verbal explanations were elicited.

Stimuli: Two special agents (God, deceased human) were included in the stimuli but not reported here as they are not relevant to the current questions. Stimuli also included a frog in the US but not in Panama (only 2 Ngöbe participants saw the frog), so this item is excluded from ranking results. (Note: Four US interviews had either or both rain and rock as missing items. These were treated as missing but the rank order still included them in the tie for last.)

Rankings: To keep interviews of reasonable duration, each participant ranked 2-4 capacities. The number of capacities ranked did not differ significantly across groups (Ngöbe $\mathrm{M}=2.81$, US $\mathrm{M}=3.18, \mathrm{t}(20)=-1.05$, $n s)$. Each capacity was ranked by a similar number of Ngöbe and U.S. respondents, $\chi 2(1, N=22)$ from 0.00 to 1.70 for each capacity, all ns. The selection of and order for the capacity probes was random, except that communication usually appeared first in Panamanian interviews because pilot work suggested its centrality for Ngöbe. (Note: 8 of 11 Ngöbe saw Communication first; 4 of 11 US students saw Communication first. This difference in distribution is not significant. The other 4 capacities were presented in random order for both groups.)

Explanation probes. Participants generally provided one explanation per entity (e.g., chimp) or kind (e.g., all animals), with number of explanations provided for a given capacity ranging from 2 to 19 . Average length of explanation did not vary by culture (Ngöbe $\mathrm{M}=11.17$ words, $\mathrm{SD}=2.30$; $\mathrm{US} \mathrm{M}=12.04$ words, $\mathrm{SD}$ $=2.01, \mathrm{t}(20)=.-.94, \mathrm{~ns})$; nor did the average number of explanations provided by individual participants (Ngöbe $\mathrm{M}=20.18, \mathrm{SD}=10.19$; US $\mathrm{M}=19.64, \mathrm{SD}=8.39, \mathrm{t}(20)=.14, \mathrm{~ns}$ ).

\section{Bilingual protocol}

Probe stem, for Communication: I will ask you to decide which thing is more capable of [conveying thoughts or feelings to others]. Please look at all the things here, then rank them in order of top to bottom, from those who are most capable of [conveying thoughts or feelings to others], to those who are least capable." Voy a pedirle que decida, ¿Cual cosa es más capaz de [expresar pensamientos o sentimientos a los demás]? Por favor, mire a todos los cosas aquí, y ordénelos desde los más capaces [de expresar pensamientos o sentimientos a los demás] hasta los menos capaces.

Desire. Which thing is more capable of longing or hoping for things? ¿Cual cosa es mas capaz de desear o esperar las cosas?

Thought. Which thing is more capable of thinking? ¿Cual cosa es mas capaz de pensar?

The remaining bilingual probes were identical to the Protocol used in Appendix B2 (without the relational condition). 


\section{A2. Detailed results: Agency attributions}

Other effects. As expected, there were also significant main effects of culture and kind on both inclusions and rankings. Overall, Ngöbe included more entities in their agency ranks $(M=0.66, S D=0.20)$ than did US participants $(M=0.45, S D=0.15), F(1,20)=7.82, p<.05, \eta^{2}=.28$. Overall mean ranks also differed slightly (Ngöbe: $M=8.88, S D=0.22$; US: $M=8.66, S D=0.16$ ), $F(1,20)=6.69, p<.05, \eta^{2}=.25$. They are not exactly equal across groups because mean ranks are aggregated by kind, leading to a weighted mean rank. Overall mean rankings are identical when averaged across the 16 items individually for an unweighted average of 8.5. There were main effects for kind on both inclusions, $F(3.24,64.87)=61.48, p<$ $.01, \eta^{2}=.76$, and rankings, $F(3.47,69.35)=114.41, p<.01, \eta^{2}=.85$. Humans and animals were included more than the other three kinds, ( $p s<.05)$, with humans ranked highest followed by animals $(p s<.05)$, and then the three non-animal kinds, which did not differ from one another (these differences are noted with subscripts in Table 1 in the main text).

\section{A3. Coding scheme for explanations}

Table A1: Inter-rater reliabilities for coding variables

\begin{tabular}{|l|cc|}
\hline \multicolumn{2}{|l|}{ Inter-rater reliability tests } \\
\hline Coding variable & Alpha & ICC \\
\hline Framings of Agency & & \\
Human-centric framing & 0.81 & 0.69 \\
Scalar framing & 0.81 & 0.69 \\
Social relations framing & 0.92 & 0.85 \\
Ecological relations framing & 0.88 & 0.79 \\
& & \\
Agency Constructs & & 0.76 \\
Internalized indicators & 0.86 & 0.70 \\
Interactive indicators & 0.83 & 0.77 \\
Consciousness criteria & 0.87 & 0.62 \\
Directedness criteria & 0.77 & 0.79 \\
Individual Processes & 0.88 & \\
\hline
\end{tabular}

* All ICC tests are significant, $\mathrm{p}<.01$, with 232 degrees of freedom

\section{A4. Detailed Results: Explanatory frameworks for agency}

Explanation corpus: The final data set included a total of 429 explanations from 20 participants across the five agency capacities and five kinds. Agency capacities: The cultural distribution of explanations provided for each capacity did not vary by culture (overall explanations per capacity: Thought, 64; Communication, 151; Morality, 62; Hunger, 79; Desire, 73). Entity kind: The number of explanations provided for each kind did not differ by culture. There was a reliable main effect of kind, $\mathrm{F}(1,18)=15.77$, $\mathrm{p}<.01, \eta^{2}=.47$, with more explanations provided for animate kinds $(\mathrm{M}=14.20, \mathrm{SD}=6.40)$ than inanimate kinds $(\mathrm{M}=7.25, \mathrm{SD}=5.14)$. There was no interaction of culture by kind.

Coding capture. Coding capture refers to the portion of the total explanatory content provided by individuals that was captured by the coding scheme. There was no main effect of culture on coding 
capture (Ngöbe $\mathrm{M}=18.20 \%$, $\mathrm{SD}=5.10 \%$; US M $=16.07 \%$, SD $=3.41 \%, \mathrm{t}(18)=1.10$, ns), indicating that the coding scheme successfully captured modes of reasoning relevant to both groups.

Detailed results for key coding constructs.

Relational framings of agency: Broken down by social and ecological relations. Overall, Ngöbe relied on relational framings of agency (social + ecological) significantly more often than US participants. The same cultural trend held separately for social relations, $\mathrm{F}(1,18)=4.78, \mathrm{p}<.05, \eta^{2}=.21$, and non-significantly for ecological relations, $\mathrm{F}(1,18)=2.95, \mathrm{p}=.10, \eta^{2}=.14$. Our coding system followed the Western sense of "social" so the distinction between social and ecological depended on the grammatical subjects (animate or inanimate). Thus, it is unsurprising that there was a main effect of kind $\mathrm{F}(1,18)=6.50, \mathrm{p}<.05, \eta^{2}=.27$, such that explanations for animate kinds had more social relational content $(\mathrm{M}=3.52 \%, \mathrm{SD}=3.13 \%)$ than explanations for inanimate kinds $(\mathrm{M}=1.62 \%, \mathrm{SD}=2.15 \%)$. And vice versa, inanimate explanations had more ecological relational content $(\mathrm{M}=5.26 \%, \mathrm{SD}=4.29 \%)$ than animates $(\mathrm{M}=.90 \% \mathrm{SD}=1.47 \%), \mathrm{F}(1,18)=$ $19.79, \mathrm{p}<.01, \eta^{2}=.52$. No interactions of culture by kind were found for either category.

\section{Appendix B: Experiment 2}

\section{B1. Methods and procedure}

Items: Simple artifact: In Panama, the simple artifact was a handmade net bag. In the US, it was a standard (industrially-manufactured) tote bag. This difference between the two artifacts had no discernable effects on results-capacity attributions to the bag were uniformly low for both Ngöbe and US participants.

Counterbalanced probe orders: (Order A) Move, morality, fear, memory, communication, hunger, pain, live; (Order B) Move, pain, hunger, communication, memory, fear, morality, live.

Experimental condition: In the relational condition, each capacity probe identified an object (or objects) of the agency predicate (e.g., can $x$ feel hunger for food or nutrients?). Each probe was always presented with the focal objects noted in the protocol below; other objects were occasionally used if further examples were needed. Every participant in the relational condition heard the initial probe for a capacity in the relational format. However, the total number of relational probes given by the experimenter during the rest of the ranking process varied across participants due to interview dynamics (e.g., some participants sorted rapidly, requiring fewer experimenter probes overall and thus receiving fewer relational probes).

Demographic items: Religiosity: In addition to reporting church attendance, participants responded to three belief questions drawn from the original mind perception survey of Gray et al. 2007: 1) I consider myself to be strongly religious [or spiritual] / Yo me considero muy religioso [o espiritual]; 2) I believe that God exists / Yo creo que Dios existe; 3) I believe that people whose bodies are dead continue to live on spiritually / Yo creo que las personas cuyos cuerpos están muertos, siguen viviendo espiritualmente. Experience with nature: In addition to reporting their place of primary residence in childhood (urban, suburban, rural), participants responded to the following questions about experience with nature: 1) Do you [your parents] have a farm or garden? / ¿Tiene [n] [sus parientes] una finca o un jardín? 2) In general, I would say that my experience with planting, farming, or harvesting is: [5-point response scale] / En general, yo diría que mi experiencia con la siembra, la finca, o la cosecha es: 2) In general, I would say that my experience with hunting and fishing is: / En general, yo diría que mi experiencia con la caza y la pesca es: (same scale) 3) Do you have a pet now [have you ever in your life]? ¿Tiene una mascota, ahora [en tu vida]? 


\section{B2. Bilingual protocol}

Instructions: In this task, we will ask you different kinds of questions. There are no right or wrong answers; please respond with your thoughts.

En esta tarea, te vamos a preguntar diferente tipos de preguntas. No hay respuestas correctas ni incorrectas. Puedes responder como piensas.

Practice task: Okay, let's start by practicing some questions to see the task goes. First, we'll practice ranking. The example is size. So the question is: Decide which of these is the biggest.

Vamos a empezar con unas preguntas de practica para ver cómo va ser la tarea. Primero vamos a practicar clasificación. El ejemplo para practicar es el tamaño. Entonces, la pregunta es: ¿Cuáles cosas son más grande?

\section{Bilingual Questions, for Original \& [Relational] Conditions}

General question stem: Which things are most capable of $[X]$ ? Please consider each thing and rank them in order of top to bottom, from those who are most capable of [X], to those who are least capable. If some of them are equal, you can put them together in the same row. If some of them don't have any capacity to do $[\mathrm{X}]$ at all, you can leave them out.

¿Cuáles cosas son mas capaz de [x]? Por favor, mira todas las cosas aquí, y ordénelas desde arriba hacia abajo las que son más capaces de [x] hasta las menos capaces. Si algunas son iguales, puedes ponerlas en el mismo puesto. Si algunas de ellas no tienen ningún capaz de [x], puedes ponerlas afuera.

(i) Move: Which things are most capable of moving? [Same across both conditions]

Cuales de las cosas son mas capaz de moverse? [Same across both conditions]

1. Morality: Which things are more capable of telling right from wrong (or good and bad) and trying to do the right (or wrong) thing? [Relational condition: to others (of their kind)?]

Bueno y Malo: ¿Cuáles cosas son mas capaz de reconocer lo bueno y lo malo y de tratar de hacer lo bueno (o lo malo) [Relational condition: a los demás (entre ellos)?]

2. Fear: Which things are more capable of feeling afraid or fearful? [Relational condition: e.g., of enemies or plagues?]

Temor: ¿Cuales cosas son mas capaz de sentir temor o miedo? [Relational condition: e.g., de los enemigos, or las plagas?]

3. Memory: Which things are more capable of remembering things? [Relational condition: e.g., where food, water, or their home/nest is?]

Memoria: ¿Cuales cosas son mas capaz de recordar las cosas? [Relational condition: e.g., donde está su comida, agua, o su hogar/nido?]

4. Communication: Which things are more capable of expressing feelings? [Relational condition: to others (of their kind)? $]^{30}$

\footnotetext{
30 The original mind perception survey item read "conveying thoughts or feelings to others," but this probe has two predicates that may tap different conceptions, so we chose a single predicate (presumably the more inclusive one) with "conveying feelings to others."
} 
Comunicación: ¿Cuáles cosas son más capaz de expresar sentimientos? [Relational condition: a los demás (entre ellos)?]

5. Hunger: Which things are more capable of feeling hungry? [Relational condition: e.g., for food or nutrients?]

Hambre: ¿Cuales cosas son mas capaz de sentir hambre [Relational condition: e.g., por comida o nutrientes?]

6. Pain: Which things are more capable of feeling physical pain? ${ }^{31}$ [Relational condition: e.g., from being hurt or sick?]

Dolor: ¿Cuáles cosa son mas capaz de sentir dolor físico [Relational condition: e.g., de ser lastimado o enfermo?]

(ii) Live: Which things have more capacity to be alive? [Same across both conditions]

Vivir: Cuales cosas son mas capaz de estar vivos?

\section{B3. Participants}

Don't know responses. These were treated as missing data. For inclusions, this was appropriate given that composite variables (by kind and dimension) reflect a simple mean. For the weighted rank system, however, too many missing data points led to ranks that were not strictly comparable across participants. This is because the weighted rank for each participant was calculated from the total number of items ranked, such that the lowest item (an exclusion or a last place rank) would be ranked "12" for a participant who ranked only 12 entities (leaving out two 'don't knows') but "14" for one who did rank all the entities (regardless of whether all 14 were included or not). Most Ngöbe participants (6 of 8) who gave a "don't know" response did so only once (for a single item on a single capacity ranking), so it did not appreciably affect their aggregated ranks by kind or dimension. However, two Ngöbe participants systematically gave "don't know" responses for the sun and the ocean across multiple capacities, so their aggregate rank scores did not fit the $[1,14]$ scale and were excluded from analysis.

\section{B4. Detailed results for preliminary analyses}

Probe order. The six agency capacities were presented in one of two counterbalanced orders, the same for both cultural groups. A series of analyses on the focal outcome measures (with culture and task order as between-subjects factors) confirmed that there was no effect of probe order on agency attributions, as measured by rates of inclusion. Similarly, there was no effect of probe order on mean ranks among Ngöbe. However, probe order did affect mean ranks for US participants: the place of plants versus complex artifacts is flipped across the two probe orders, and this is reflected most strongly on the cognition dimension (there is no effect of probe order for experience). US participants were more likely to rank complex artifacts above plants on the probe order that presented Morality first, as compared to when Pain was presented first. This effect was of small enough magnitude that the overall cultural attribution profile still ranked complex artifacts well above plants on the cognition dimension (as reported in the main text).

1560

\footnotetext{
${ }^{31}$ The original mind perception survey item read "experiencing physical or emotional pain," but this probe has two
} parts so we again chose a single predicate (presumably the more inclusive one) with "feeling physical pain." 


\section{B5. Detailed results for focal analyses}

Overall analysis: As expected, there was a main effect of culture for inclusions, $F(1,54)=19.61, \mathrm{p}<.001, \eta^{2}$ $=0.27$, such that Ngöbe had higher rates of inclusion overall $(M=.53, S D=.14)$ than US participants $(M=$ $.44, S D=.06)$. This cultural main effect was moderated by kind and (separately by) condition and dimension as described in the main text. There was no main effect of culture for ranks.

\section{Cultural models of agency}

Cultural agency attribution profiles by mean rank

Appendix Table B1. Mean ranks for agency ${ }^{\mathrm{a}}$ by culture

\begin{tabular}{|c|c|c|c|c|c|c|c|}
\hline \multirow[b]{3}{*}{ Kind $^{c}$} & \multicolumn{7}{|c|}{ Mean ranks ${ }^{b}$} \\
\hline & \multicolumn{2}{|c|}{$\begin{array}{l}\text { Ngöbe } \\
(n=22)\end{array}$} & \multicolumn{2}{|c|}{$\begin{array}{c}\text { US } \\
(n=35)\end{array}$} & \multirow[b]{2}{*}{$U$-test } & \multicolumn{2}{|c|}{$\begin{array}{l}\text { Overall } \\
(N=57)\end{array}$} \\
\hline & $M$ & $S D$ & $M$ & $S D$ & & $M$ & $S D$ \\
\hline Humans & $2.90_{a}$ & $(0.91)$ & $2.87 \mathrm{a}$ & $(0.62)$ & $n s$ & $2.88_{a}$ & $(0.73)$ \\
\hline Animals & $5.04_{\mathrm{b}}$ & $(0.98)$ & $4.28_{\mathrm{b}}$ & $(0.39)$ & $184.5^{* *}$ & $4.57_{\mathrm{b}}$ & $(0.77)$ \\
\hline Plants & $8.36_{c}$ & $(1.29)$ & $9.39_{c}$ & $(0.89)$ & $172^{* * *}$ & $8.99_{c}$ & $(1.17)$ \\
\hline Abiotic kinds & $9.49_{d}$ & $(1.47)$ & $11.01_{\mathrm{d}}$ & $(0.31)$ & $72.5^{* * *}$ & $10.42_{\mathrm{d}}$ & $(1.19)$ \\
\hline Complex artifacts & $10.99 \mathrm{e}$ & $(0.70)$ & $9.61_{\mathrm{c}}$ & $(1.12)$ & $92.5^{* * *}$ & $10.14_{\mathrm{d}}$ & $(1.19)$ \\
\hline Simple artifacts & $11.63_{f}$ & $(0.76)$ & $11.13_{\mathrm{e}}$ & $(0.35)$ & $235.5^{*}$ & $11.32_{\mathrm{e}}$ & $(0.60)$ \\
\hline
\end{tabular}

${ }^{a}$ Agency is a composite measure of memory, communication, morality, hunger, pain, and fear.

${ }^{\mathrm{b}}$ Lower numbers indicate higher ranks (1 - 14); excluded items scored as tied for last.

${ }^{\mathrm{c}}$ Main effect for kind: Means in the same column not sharing the same subscript differ significantly at $p<.05$ in Wilcoxon signed-rank tests.

Note: ${ }^{*}$ Indicates $p<.05 ;{ }^{* *} p<.01 ;{ }^{* *} p<.001$.

\section{Experimental Condition}

Experimental Condition: Inclusions. There was a main effect of condition, $F(1,54)=10.08, p<.01, \eta 2=0.16$, with higher rates of inclusion in the relational condition $(M=.50, S D=.14)$ as compared to the original condition $(M=.45, S D=.05)$. But this main effect of condition was moderated by culture, such that agency attributions only varied by condition for Ngöbe participants.

Details for US inclusions by experimental condition: Original: $M=.45, S D=.04$; Relational: $M=.43, S D=.07$; condition: $F(1,33)=1.16, p=.29, \eta^{2}=.03$; condition by kind, $F(2.22,73.25)=1.58, p=.21, \eta^{2}=0.05$.

\section{Conceptual organization of agency concepts}

Correlations. We also conducted a series of pairwise correlations between each of the eight individual capacities for each kind, considering each cultural group separately. These results told a similar story to that of the aggregate conceptual dimensions reported in the main text.

\section{B6. Cultural Consensus Modeling}


1592 Technically, ranking data (as opposed to normal continuous data) violate the axiom of conditional 1593 independence given that a response for one item influences the ranks available for another item. In such a 1594 case, the posterior checks can be considered approximations rather than exact indicators. However, this 1595 does not mean the model used is problematic. In fact others have used a Thurstonian model for rankings 1596 identical to the method used here (Lee et al., 2012; Lee et al., 2014), except that the CRM, which detects 1597 latent truths, extends this model to multiple cultures and also accounts for response biases and item 1598 difficulty. Additionally, the more ranks one uses (e.g., items) the less severe the violation of conditional 1599 independence; hence, we used the item-level data with 14 items rather than kind-level data with 5 kinds. 1600 As one final note, CCTpack includes two participant response bias parameters as a default setting (this 1601 measures the tendency to use the extremes as opposed to the middle of the scale). We did not include 1602 response bias parameters in our modeling simulations due to constraints from too many degrees of 1603 freedom. 